\title{
The homology of real subspace arrangements
}

\author{
Eric M. Rains
}

\begin{abstract}
Associated to any subspace arrangement is a 'De Concini-Procesi model', a certain smooth compactification of its complement, which in the case of the braid arrangement produces the Deligne-Mumford compactification of the moduli space of genus 0 curves with marked points. In the present work, we calculate the integral homology of real De Concini-Procesi models, extending earlier work of Etingof, Henriques, Kamnitzer and the author on the (2-adic) integral cohomology of the real locus of the moduli space. To be precise, we show that the integral homology of a real De Concini-Procesi model is isomorphic modulo its 2-torsion to a sum of cohomology groups of subposets of the intersection lattice of the arrangement. As part of the proof, we construct a large family of natural maps between De Concini-Procesi models (generalizing the operad structure of moduli space), and determine the induced action on poset cohomology. In particular, this determines the ring structure of the cohomology of De Concini-Procesi models (modulo 2-torsion).
\end{abstract}

\section{Contents}

1. Introduction

2. De Concini-Procesi models

3. Poset homology and operad maps

4. Blow-ups and homology

5. A cell structure for $\bar{Y}_{\mathcal{G}}$

6. 2-adic homology

7. Operadicity

8. Further directions

References

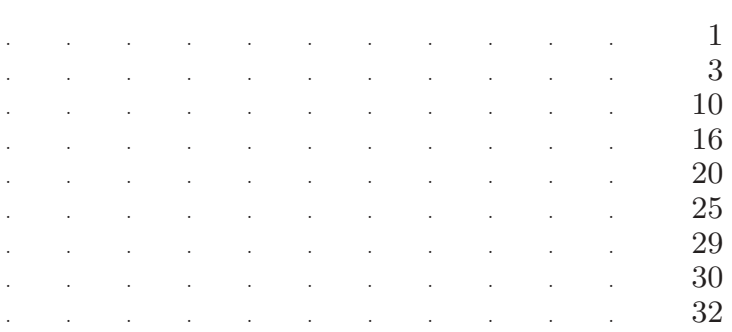

\section{Introduction}

The main result of [7] was the determination of the cohomology ring structure of the real locus $\overline{M_{0, n}}(\mathbb{R})$ of the moduli space of stable genus 0 curves with $n$ marked points. It was shown there that the cohomology of $\overline{M_{0, n}}(\mathbb{R})$ could be expressed in terms of the homology of intervals of a certain poset, namely the poset of partitions of $\{1,2, \ldots, n-1\}$ in which each part is of odd size. This was, of course, quite reminiscent of the corresponding result (see, for example, $[\mathbf{1 5}]$ ) for the cohomology of the configuration space of $n$ distinct points in $\mathbb{P}^{1}(\mathbb{C})$, which is expressed in terms of the homology of the poset of all partitions of $\{1,2, \ldots, n-1\}$. As the latter result generalizes to arbitrary subspace arrangements $[\mathbf{5}, \mathbf{1 0}, \mathbf{1 6}, \mathbf{1 9}]$, it was natural to look for a corresponding generalization of $[\mathbf{7}]$.

In our context, the appearance of subspace arrangements comes from an interpretation of $\overline{M_{0, n}}$ (real or complex) as a special case of a construction of De Concini and Procesi [4]

Received 10 December 2009.

2000 Mathematics Subject Classification 14N20 (primary), 14F25 (secondary).

The author was supported in part by NSF Grant No. DMS-0401387. 
of a 'wonderful' compactification associated to an arbitrary subspace arrangement (in this case, the braid arrangement of type $A_{n-2}$, as remarked in [4, p. 483]). When the spaces in the arrangement are real, the De Concini-Procesi model is a smooth real projective variety, and thus gives rise to a smooth manifold. In the present note, we determine the (integral) homology groups of these manifolds. More precisely, the homology of these manifolds consists of a large amount of 2-torsion (analogous to the homology of the spaces $\mathbb{R} \mathbb{P}^{n}$ ), which is implicitly determined (via the mod 2 homology) in Section 6 below, but if we quotient out by this 2 -torsion, the result can then be expressed (canonically) as a direct sum of cohomology groups of certain simplicial complexes described in Section 3. These simplicial complexes can in turn be subdivided to obtain the order complexes of certain subposets of the lattice of subspaces in the arrangement, the primary constraint (2-divisibility) on the subspaces being that they decompose as transverse intersections of even-codimensional subspaces. The precise statement of the main theorem is given as Theorem 3.7 below.

As might be guessed from the fact that we switched to considering homology rather than cohomology as in [7], our techniques are somewhat different; in particular, we do not have the luxury of an explicit presentation for the cohomology algebra. (Similarly, while [7] made some use of the fact that $\overline{M_{0, n}}(\mathbb{R})$ is a $K(\pi, 1)$, this fails to hold for arbitrary real De ConciniProcesi models (for example, $\mathbb{R} \mathbb{P}^{n}$ ).) The approach of $[\mathbf{7}]$ of obtaining information about 2-adic (co)homology using the mod 2 (co)homology does play a significant role, however (see Section 6 below). Moreover, one of the main tools of [7] was the fact that the moduli spaces form an operad, giving rise to a large collection of natural maps that were used there to distinguish cohomology classes; one of our main tools (even more so than in $[\mathbf{7}]$ ) is the observation that these operad maps can be defined for general De Concini-Procesi models. In particular, this includes diagonal morphisms; thus, although we work exclusively with homology, we still effectively (if somewhat implicitly) determine the ring structure on cohomology.

Our primary remaining tool, which was not used in [7], is a certain long exact sequence associated to blow-ups of real varieties. Since every De Concini-Procesi model is a blow-up of a simpler De Concini-Procesi model, this allows us to reduce a significant portion of the main theorem (localized away from the prime 2) to the (trivial) case of products of projective spaces. In particular, this allows us to resolve a conjecture of [7] by showing that the (co)homology of $\overline{M_{0, n}}(\mathbb{R})$ has no odd torsion. This holds for arbitrary Coxeter arrangements (see [11]) but again not for general De Concini-Procesi models. (In fact, even if one restricts one's attention to hyperplane arrangements, one can arrange for the homology of an arbitrary finite simplicial complex to appear as a graded piece of the homology of some De Concini-Procesi model; see the remark following the statement of the main theorem.)

The plan of the paper is as follows. In Section 2, we recall De Concini and Procesi's construction, and introduce the associated 'operad' maps (together with the main 'composition law' that they satisfy); we also show how these maps give rise to a natural grading of the homology groups by the lattice of subspaces in the arrangement. In Section 3, we introduce the corresponding combinatorial data, in particular allowing us to state the main theorem (Theorem 3.7). The final set-up section, Section 4, establishes the long exact sequence associated to a real blow-up. Corollary 4.5 of this section discusses the special case of the blow-up long exact sequence associated to De Concini-Procesi models, and in particular shows how this sequence interacts with the natural grading by subspaces.

The proof of the main theorem spans Sections 5-7. In Section 5, we construct a family of natural cell structures on the De Concini-Procesi model, which allow us to construct the isomorphism of the main theorem via a chain map. It is then fairly straightforward to show that the chain map respects the blow-up long exact sequence, and thus by induction that it induces an isomorphism on homology with coefficients in $\mathbb{Z}[1 / 2]$. This argument fails to control the $2^{k}$-torsion, however, which is the subject of Section 6 ; there, we show that the chain map gives an isomorphism (modulo 2 -torsion) over $\mathbb{Z} / 4 \mathbb{Z}$, which then implies the isomorphism property 
over the 2-adic integers. In the process, we give an explicit basis of the mod 2 homology consistent with the natural grading (its primary distinction from the cohomology basis of [18]), which together with the main theorem determines the 2-torsion of the homology groups. The proof of the main theorem is finished in Section 7, where we show that the action of the operad maps on homology agrees with the action described combinatorially in Section 3. This in particular determines the ring structure on cohomology.

Finally, in Section 8, we discuss some possibilities for further generalizations; see especially Conjecture 1, which considers the case of $\mathbb{R}$-rational (that is, closed under conjugation) arrangements. Also of some interest is Theorem 8.1, which gives an interpretation of the cohomology of the full poset of '2-divisible' subspaces (when that poset has no maximal element) in terms of the homology, twisted by a certain sheaf, of the De Concini-Procesi model.

Notational CONVEntion. Unless otherwise stated, all modules are supermodules, with corresponding conventions for tensor products.

\section{De Concini-Procesi models}

We will need to recall (and extend) some notions from [4]. Note that our definition is slightly more general than theirs, but only in that their construction is not closed under taking products (see Lemma 2.3 and surrounding remarks). The considerations of functoriality and operadicity are new, and in particular the construction of a natural grading by subspaces on the (co)homology groups (see the remark following Corollary 2.8).

A subspace arrangement in a finite-dimensional vector space $V$ is a finite collection $\mathcal{G}$ of subspaces of $V^{*}$. Note that this induces by duality a corresponding collection of subspaces of $V$, but it will be convenient to use the dual notation. Given a subspace arrangement $\mathcal{G}$, we define $\mathcal{C}_{\mathcal{G}}$ to be the lattice generated by $\mathcal{G}$; that is, the set of all sums of subsets of $\mathcal{G}$. (By convention, this includes the empty sum; that is, $0 \in \mathcal{C}_{\mathcal{G}}$.) Note that $\mathcal{C}_{\mathcal{G}}$ is indeed a lattice with respect to inclusion, but the meet operation in this lattice is not simply the intersection of subspaces.

Given $U \in \mathcal{C}_{\mathcal{G}}$, a $\mathcal{G}$-decomposition of $U$ is a collection of nonempty subspaces $U_{i} \in \mathcal{C}_{\mathcal{G}}$ such that

$$
U=\bigoplus_{1 \leqslant i \leqslant k} U_{i}
$$

and for every $G \in \mathcal{G}$ such that $G \subset U, G \subset U_{i}$ for exactly one $i$. Note that every element of $\mathcal{G}$ is $\mathcal{G}$-indecomposable, and the notion of decomposition depends only on the collection of $\mathcal{G}$-indecomposable subspaces. In particular, if we let $\overline{\mathcal{G}}$ denote that collection, then $\mathcal{C}_{\mathcal{G}}=\mathcal{C}_{\overline{\mathcal{G}}}$ and an element is $\mathcal{G}$-indecomposable if and only if it is $\overline{\mathcal{G}}$-indecomposable. A building set is a subspace arrangement $\mathcal{G}$ such that $\mathcal{G}=\overline{\mathcal{G}}$; we have thus seen that every arrangement induces a building set.

If $\mathcal{G}$ is a building set in $V$, and $\mathcal{G}^{\prime}$ is a building set in $V^{\prime}$, then a morphism from $\mathcal{G}$ to $\mathcal{G}^{\prime}$ is a linear transformation $f: V \rightarrow V^{\prime}$ such that $f^{*}(G) \in \mathcal{G}$ for all $G \in \mathcal{G}^{\prime}$. We thus obtain for every field $K$ a category Build $(K)$ of building sets of vector spaces over $K$. There are two important special cases of morphisms. First, if $\mathcal{G} \supset \mathcal{G}^{\prime}$ are both building sets in $V$, then the identity map on $V$ induces a morphism $\iota: \mathcal{G} \rightarrow \mathcal{G}^{\prime}$. Second, we have morphisms $f$ such that

$$
\begin{aligned}
\mathcal{G} & =\overline{\left\{f^{*}(G): G \in \mathcal{G}^{\prime}\right\}}, \\
\mathcal{G}^{\prime} & =f_{*}(\mathcal{G}):=\left\{C: C \in \mathcal{C}_{\mathcal{G}^{\prime}} \mid f^{*}(C) \in \mathcal{G}\right\} .
\end{aligned}
$$


Since, for a general morphism,

$$
\begin{aligned}
\mathcal{G} & \supset \overline{\left\{f^{*}(G): G \in \mathcal{G}^{\prime}\right\}}, \\
\mathcal{G}^{\prime} & \subset f_{*}(\mathcal{G}), \\
\overline{\left\{f^{*}(G): G \in \mathcal{G}^{\prime}\right\}} & =\overline{\left\{f^{*}(G): G \in f_{*}(\mathcal{G})\right\}},
\end{aligned}
$$

it follows that any morphism can be decomposed as $\iota \circ f \circ \iota^{\prime}$ with $f$ satisfying (2.1) and (2.2). Both types of morphisms can be further decomposed; indeed, it suffices to take morphisms with $\operatorname{dim}(\operatorname{ker} f)=1$ and $\mathcal{G}^{\prime}=f_{*}(\mathcal{G})$, and morphisms $\iota$ such that $\left|\mathcal{G}^{\prime}\right|=|\mathcal{G}|-1$. The only nontrivial thing to prove is the following.

Lemma 2.1. Given any two building sets $\mathcal{G} \supset \mathcal{G}^{\prime}$ on the same space, there exists an element $G \in \mathcal{G} \backslash \mathcal{G}^{\prime}$ such that $\mathcal{G} \backslash\{G\}$ is a building set.

Proof. Let $G$ be a minimal element of $\mathcal{G}$ not in $\mathcal{G}^{\prime}$, and follow the proof of [4, Proposition $2.5(1)]$.

De Concini and Procesi associate a smooth projective variety to a building set $\mathcal{G}$ as follows. Let $\mathcal{A}_{\mathcal{G}}$ be the affine variety

$$
\mathcal{A}_{\mathcal{G}}=V \backslash \bigcup_{G \in \mathcal{G}} G^{\perp}
$$

Then for each $G$ we have a map $\mathcal{A}_{\mathcal{G}} \rightarrow \mathbb{P}_{G}$, where $\mathbb{P}_{G}=\mathbb{P}\left(V / G^{\perp}\right)$, and we thus have a map

$$
\mathcal{A}_{\mathcal{G}} \longrightarrow \prod_{G \in \mathcal{G}} \mathbb{P}_{G}
$$

Then $\bar{Y}_{\mathcal{G}}$ is the closure of the image of this map. A more local description can be given as follows: let $\rho_{G}: \bar{Y}_{\mathcal{G}} \rightarrow \mathbb{P}_{G}$ be the natural map. Then $\bar{Y}_{\mathcal{G}}$ is the locus of points $x \in \prod_{G \in \mathcal{G}} \mathbb{P}_{G}$ such that for every pair $H \subset G \in \mathcal{G},\left(\rho_{G}(x), \rho_{H}(x)\right)$ is in the (closed) graph of the projection $\mathbb{P}_{G} \rightarrow \mathbb{P}_{H}$.

If $\operatorname{dim}(G)=1$ (that is, if $G^{\perp}$ is a hyperplane), then $\mathbb{P}_{G}$ is a single point, so we find that removing all hyperplanes from a building set has no effect on the corresponding variety. Conversely, adjoining a hyperplane to a building set has no effect, so long as the result is still a building set. A useful criterion for this is the following.

Lemma 2.2. Suppose $v \in V^{*}$ is a nonzero vector such that for any $C \in \mathcal{C}_{\mathcal{G}}$ containing $v$ there exists $G \in \mathcal{G}$ such that $v \in G \subset C$. Then $\mathcal{G} \cup\{\langle v\rangle\}$ is a building set.

Proof. Let $\mathcal{G}^{\prime}:=\mathcal{G} \cup\{\langle v\rangle\}$, and consider $C \in \mathcal{C}_{\mathcal{G}^{\prime}}$ which is $\mathcal{G}^{\prime}$-indecomposable. We need to show that $C \in \mathcal{G}^{\prime}$.

If $v \notin C$, then $C \in \mathcal{C}_{\mathcal{G}}$. A $\mathcal{G}$-decomposition of $C$ would then also decompose $C$ as an element of $\mathcal{C}_{\mathcal{G}^{\prime}} ;$ it follows that $C \in \mathcal{G}$.

If $v \in C$, but still $C \in \mathcal{C}_{\mathcal{G}}$, then by assumption, there exists $G \in \mathcal{G}$ with $v \in G \subset C$. In particular, $G$ is contained in some component of the $\mathcal{G}$-decomposition of $C$, and thus $v$ is contained in that component. It follows that the $\mathcal{G}$-decomposition of $C$ is the same as its $\mathcal{G}^{\prime}$-decomposition, and thus $C \in \mathcal{G}$.

Finally, we have the case $v \in C, C \notin \mathcal{C}_{\mathcal{G}}$. In particular, we can write $C=D+\langle v\rangle$ for some $D \in \mathcal{C}_{\mathcal{G}}$ not containing $v$. We claim that the $\mathcal{G}^{\prime}$-decomposition of $C$ is obtained by adjoining $\langle v\rangle$ to the $\mathcal{G}$-decomposition of $D$. Suppose otherwise that there exists $G^{\prime} \in \mathcal{G}$ such that $G^{\prime} \subset C$ but $G^{\prime} \not \subset D$. But then $C=G^{\prime}+D \in \mathcal{C}_{\mathcal{G}}$, a contradiction. It follows that $C=\langle v\rangle$. 
In particular, if $v \in G \in \mathcal{G}$ is such that if $v \in C \in \mathcal{C}_{\mathcal{G}}$, then $G \subset C$, then $\mathcal{G} \cup\{\langle v\rangle\}$ is a building set. Over an infinite field, there exists such a vector in any $G \in \mathcal{G}$, and we may thus repeatedly adjoin hyperplanes to $\mathcal{G}$ without affecting the variety, until each $G \in \mathcal{G}$ is the span of $\operatorname{dim}(G)+1$ hyperplanes in the collection, and those hyperplanes are in general position. Letting $\mathcal{H}$ denote this collection of hyperplanes, we find $\overline{\mathcal{H}}=\mathcal{G} \cup \mathcal{H}$, and thus $\bar{Y}_{\mathcal{G}} \cong \bar{Y}_{\overline{\mathcal{H}}}$. In other words, every De Concini-Procesi model is isomorphic to the De Concini-Procesi model of a hyperplane arrangement. Thus by considering general building sets, we do not in fact add any more generality than if we merely considered the hyperplane case; they do, however, form a useful tool.

We note that the construction of $\bar{Y}_{\mathcal{G}}$ attributed to De Concini and Procesi above is not quite the construction they give. To be precise, they also include the map $\mathcal{A}_{\mathcal{G}} \rightarrow \mathbb{P}(V)$, or equivalently in our notation assume that $V^{*} \in \mathcal{G}$, in which case $\mathcal{A}_{\mathcal{G}}$ embeds in $\bar{Y}_{\mathcal{G}}$; it will be notationally convenient to allow the slightly more general case. Our case reduces easily to the case $V^{*} \in \mathcal{G}$, as follows. Given $W \subset V^{*}$, define the restriction $\left.\mathcal{G}\right|_{W}$ by

$$
\left.\mathcal{G}\right|_{W}=\{G \in \mathcal{G} \mid G \subset W\},
$$

a building set in $W^{*}=V / W^{\perp}$. Since $\left(V / W^{\perp}\right) / G^{\perp}=V / G^{\perp}$ for $G \subset W$, we immediately obtain the following, using the local description of $\bar{Y}_{\mathcal{G}}$. The root of a building set is the maximal element $\operatorname{root}(\mathcal{G})$ of the lattice $\mathcal{C}_{\mathcal{G}}$.

Lemma 2.3. For any $W \subset V^{*}$, there exists a natural map $\bar{Y}_{\mathcal{G}} \rightarrow \bar{Y}_{\left.\mathcal{G}\right|_{W}}$. If $G_{1} \oplus G_{2} \oplus \ldots \oplus$ $G_{k}$ is the decomposition of $\operatorname{root}(\mathcal{G})$, then

$$
\bar{Y}_{\mathcal{G}} \longrightarrow \prod_{1 \leqslant i \leqslant k} \bar{Y}_{\left.\mathcal{G}\right|_{G_{i}}}
$$

is an isomorphism. In particular,

$$
\operatorname{dim} \bar{Y}_{\mathcal{G}}=\sum_{1 \leqslant i \leqslant k}\left(\operatorname{dim}\left(G_{i}\right)-1\right)=\operatorname{dim}(\operatorname{root}(\mathcal{G}))-k .
$$

REMARK. In particular, each of the factors contains the appropriate ambient space. Also, for topological purposes we note that $\bar{Y}_{\mathcal{G}}$ in our notation is homotopic to the variety $Y_{\mathcal{G}}$ (the closure with a factor $V$ added to the map) discussed in [4].

Proposition 2.4. The construction $\bar{Y}$ defines a functor from $\operatorname{Build}(K)$ to the category of smooth projective varieties over $K$.

Proof. Let $f$ be a morphism from $\mathcal{G}$ to $\mathcal{G}^{\prime}$. To specify the associated map $\bar{Y}_{f}: \bar{Y}_{\mathcal{G}} \rightarrow \bar{Y}_{\mathcal{G}^{\prime}}$, it suffices to specify $\rho_{G} \circ \bar{Y}_{f}$ for each $G \in \mathcal{G}^{\prime}$. We simply take

$$
\rho_{G} \circ \bar{Y}_{f}=\rho_{f^{*}(G)} \circ \mathbb{P}(f),
$$

where $\mathbb{P}(f): \mathbb{P}\left(V /\left(f^{*}(G)\right)^{\perp}\right) \rightarrow \mathbb{P}\left(V^{\prime} / G^{\perp}\right)$ is the natural morphism, which is well defined (and injective) since $\left(f^{*}(G)\right)^{\perp}=f^{-1}\left(G^{\perp}\right)$. The local conditions are then straightforward to verify, as is the fact that $\bar{Y}$ respects composition of morphisms.

REMARK. The defining maps $\rho_{G}$ are associated in this way to the morphisms $\iota: \mathcal{G} \rightarrow\{G\}$. Similarly, the diagonal map $\bar{Y}_{\mathcal{G}} \rightarrow \bar{Y}_{\mathcal{G}} \times \bar{Y}_{\mathcal{G}}$ is associated to the diagonal map $\Delta: V \rightarrow V \oplus V$; more precisely, the diagonal map is the composition

$$
\bar{Y}_{\mathcal{G}} \stackrel{\bar{Y}_{\Delta}}{\longrightarrow} \bar{Y}_{\mathcal{G} \oplus \mathcal{G}} \stackrel{\sim}{\longrightarrow} \bar{Y}_{\mathcal{G}} \times \bar{Y}_{\mathcal{G}} .
$$


In fact, $\bar{Y}$ satisfies a more general version of functoriality, in that it has a sort of 'operadic' structure. Let $\mathcal{G}$ and $\mathcal{G}^{\prime}$ be building sets in $V$ and $V^{\prime}$, respectively. A weak morphism $f: \mathcal{G} \rightarrow \mathcal{G}^{\prime}$ is a linear transformation $f: V \rightarrow V^{\prime}$ such that $f^{*}(G) \in \mathcal{G} \cup\{0\}$ for all $G \in G^{\prime}$.

TheOREm 2.5. Given any weak morphism $f: \mathcal{G} \rightarrow \mathcal{G}^{\prime}$, there exists a natural morphism (the operad map)

$$
\phi_{f}: \bar{Y}_{\left.\mathcal{G}^{\prime}\right|_{\operatorname{ker}\left(f^{*}\right)}} \times \bar{Y}_{\mathcal{G}} \longrightarrow \bar{Y}_{\mathcal{G}^{\prime}}
$$

These maps satisfy the composition law, which states that given any two weak morphisms $f: \mathcal{G} \rightarrow \mathcal{G}^{\prime}, g: \mathcal{G}^{\prime} \rightarrow \mathcal{G}^{\prime \prime}$, the diagram

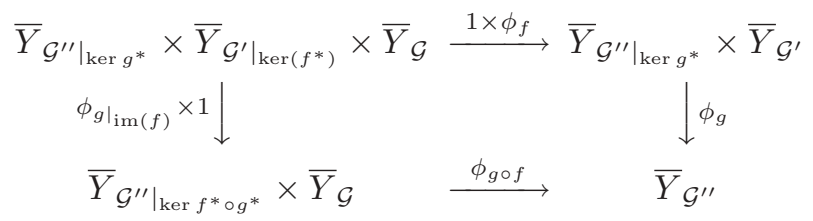

commutes, where $\left.g\right|_{\mathrm{im}(f)}$ is the induced weak morphism

$$
\left.g\right|_{\operatorname{im}(f)}:\left.\left.\mathcal{G}^{\prime}\right|_{\operatorname{ker}\left(f^{*}\right)} \longrightarrow \mathcal{G}^{\prime \prime}\right|_{\operatorname{ker}\left(f^{*} \circ g^{*}\right)}
$$

Proof. We need to specify $\rho_{G} \circ \phi_{f}$ for each $G \in \mathcal{G}^{\prime}$. If $G \subset \operatorname{ker}\left(f^{*}\right)$, then we simply set

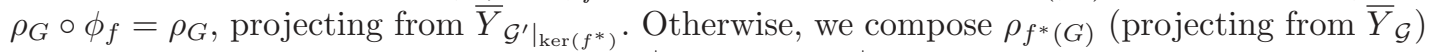
with the induced map $\mathbb{P}(f): \mathbb{P}\left(V /\left(f^{*}(G)\right)^{\perp}\right) \rightarrow \mathbb{P}\left(V^{\prime} / G^{\perp}\right)$ as before.

REMARK 1. Note that if $f$ is a linear transformation such that $\operatorname{ker}\left(f^{*}\right) \in \mathcal{C}_{\mathcal{G}^{\prime}}$, then $\left\{f^{*}(G)\right.$ : $\left.G \in \mathcal{G}^{\prime} \mid f^{*}(G) \neq 0\right\}$ is a building set in $V$, called the induced building set, and denoted by $f^{*}\left(\mathcal{G}^{\prime}\right)$. We will denote the corresponding weak morphism from $f^{*}\left(\mathcal{G}^{\prime}\right)$ to $\mathcal{G}^{\prime}$ by $\tau(f)$, and say that such a weak morphism is purely operadic. Note that the corresponding operad map is injective. For $C \in \mathcal{G}^{\prime}$, we denote by $\phi_{C}$ the operad map associated to $\tau\left(i_{C}\right)$ where $i_{C}: C^{\perp} \rightarrow V^{\prime}$ is the inclusion map.

REMARK 2. In general, any weak morphism can be factored as a product of a morphism and a purely operadic weak morphism. Indeed, given a general weak morphism $f: \mathcal{G} \rightarrow \mathcal{G}^{\prime}$, let $C=\operatorname{root}\left(\left.\mathcal{G}^{\prime}\right|_{\text {ker } f^{*}}\right)$. Then we may factor the linear transformation $f$ as $i_{C} \circ g$. But then as a weak morphism, $f=\tau\left(i_{C}\right) \circ g$. The composition law in this case simplifies, and thus we find

$$
\phi_{f}=\phi_{C} \circ\left(1 \times \bar{Y}_{g}\right)
$$

as one might expect.

REMARK 3. It will be helpful to note the forms the composition law takes when one of the maps is a morphism. If $f$ is a morphism, then

$$
\phi_{g \circ f}=\phi_{g} \circ\left(1 \times \phi_{f}\right)
$$

while if $g$ is a morphism, then

$$
\phi_{g} \circ \phi_{f}=\phi_{g \circ f} \circ\left(\phi_{\left.g\right|_{\mathrm{im}(f)}} \times 1\right) .
$$

Of course, if both are morphisms, then the composition law is simply

$$
\phi_{g \circ f}=\phi_{g} \circ \phi_{f} .
$$


Example. As an example, consider the braid arrangement

$$
A_{n-1}=\left\{\left\langle e_{i}-e_{j}\right\rangle: 1 \leqslant i<j \leqslant n\right\} .
$$

The indecomposable subspaces in this arrangement are those of the form

$$
\left\langle e_{i}-e_{j}: i, j \in S\right\rangle
$$

for some subset $S \subset\{1,2, \ldots, n\}$ with $|S| \geqslant 2$, and general subspaces are associated to partitions of $\{1,2, \ldots, n\}$. The associated De Concini-Procesi model is thus constructed as a subvariety of a product of projective spaces of the form

$$
\prod_{S \subset\{1,2, \ldots, n\}} \mathbb{P}^{|S|-2}
$$

to be precise, it is the intersection of the graphs of the maps $\mathbb{P}(S) \rightarrow \mathbb{P}(T)$ for $T \subset S$ given by omitting those coordinates not in $T$.

There is a natural morphism from the moduli space $\overline{M_{0, n+1}}$ (with one marked point singled out as special) to this De Concini-Procesi model, as follows. For each subset $S \subset\{1,2, \ldots, n\}$, we map the moduli space to $\mathbb{P}(S)$ by first forgetting all points with labels outside $S$, collapsing components as necessary to preserve stability, then further collapsing all components not containing the special point. We thus obtain a copy of $\mathbb{P}^{1}$ together with a function from $S$ to $\mathbb{P}^{1}$ that avoids the special point. Equivalently, taking the special point to $\infty$, we obtain a point in the affine space $\mathbb{A}^{|S|}$, with at least two distinct coordinates. The further isomorphisms of $\mathbb{P}^{1}$ quotient this affine space by the diagonal subspace, then by scalar multiplication, and thus we obtain a point in $\mathbb{P}^{|S|-2}$ associated to each stable curve. The local conditions are clearly satisfied, so this collection of morphisms to projective space induce a morphism to $\bar{Y} \overline{A_{n-1}}$. By considering its action on the open set $M_{0, n+1}$, we find that this morphism is birational; it is also straightforward to verify that it is bijective, and thus an isomorphism.

The moduli spaces (or, rather, their real or complex loci) form a topological operad in the usual sense, which is a special case of the above generalized operad structure. To be precise, for any composition $\alpha_{1}, \ldots, \alpha_{k}$ of $n$ with nonzero parts, there is an associated weak morphism $f_{\alpha}: A_{k-1} \rightarrow A_{n-1}$ which maps $e_{1}$ to the sum of the first $\alpha_{1}$ basis vectors, $e_{2}$ to the sum of the next $\alpha_{2}$ basis vectors, etc. The kernel of $f_{\alpha}^{*}$ is then a subspace in the arrangement, to wit the subspace associated to the set partition

$$
\left\{1,2, \ldots, \alpha_{1}\right\},\left\{\alpha_{1}+1, \ldots, \alpha_{1}+\alpha_{2}\right\}, \ldots,
$$

and $f^{*}\left(\overline{A_{n-1}}\right)=\overline{A_{k-1}}$. In particular, $f_{\alpha}$ is purely operadic, and the associated operad map is the usual operad map

$$
\overline{M_{0, \alpha_{1}+1}} \times \ldots \times \overline{M_{0, \alpha_{k}+1}} \times \overline{M_{0, k+1}} \rightarrow \overline{M_{0, n+1}}
$$

obtained by gluing the special points of the first $k$ curves to corresponding nonspecial points of the last curve. (Similarly, the 'forget a point' map is associated to a morphism $A_{n} \rightarrow A_{n-1}$.) Moreover, the usual operad axiom becomes just a special case of the general composition law. Note, however, that the cyclic operad structure of the moduli space does not seem to be compatible with its interpretation as a De Concini-Procesi model, as it does not respect the role of the special point.

Proposition 2.6. Let $f: \mathcal{G} \rightarrow \mathcal{G}^{\prime}$ be a weak morphism with $\mathcal{G} \subset f^{*}\left(\mathcal{G}^{\prime}\right)$. Then $\phi_{f}$ is injective.

Proof. Simply observe that if $f^{*}\left(G^{\prime}\right)=G$, then the map $\rho_{G^{\prime}}$ on the codomain is the composition of the map $\rho_{G}$ on the domain with an injection. 
Proposition 2.7. Let $f: \mathcal{G} \rightarrow \mathcal{G}^{\prime}$ be a surjective (weak) morphism. Then $\phi_{f}$ is surjective, and is birational if and only if $\operatorname{root}(\mathcal{G})=f^{*}\left(\operatorname{root}\left(\mathcal{G}^{\prime}\right)\right)$ and both roots have the same number of components.

Proof. The image of the restriction

$$
f: \mathcal{A}_{\mathcal{G}} \longrightarrow \mathcal{A}_{\mathcal{G}^{\prime}}
$$

is dense if $f: V \rightarrow V^{\prime}$ is surjective, and thus the corresponding map of projective varieties must be surjective; birationality then follows by comparing dimensions.

REMARK. In particular, this applies to any morphism of the form $\iota: \mathcal{G} \rightarrow \mathcal{G}^{\prime}$.

Corollary 2.8. Given a building set $\mathcal{G}$ and a space $C \in \mathcal{C}_{\mathcal{G}}$, the natural surjection

$$
\pi_{C}:=\phi_{\iota}: \bar{Y}_{\mathcal{G}} \longrightarrow \bar{Y}_{\left.\mathcal{G}\right|_{C}}
$$

has a natural homotopy class $\pi_{C}^{[-1]}$ of splittings. Moreover, these maps satisfy the identities (up to homotopy)

$$
\begin{aligned}
\pi_{C \wedge D} \circ \pi_{C} & =\pi_{C \wedge D}, \\
\pi_{C} \circ \pi_{D}^{[-1]} & \sim \pi_{C \wedge D}^{[-1]} \circ \pi_{C \wedge D}, \\
\pi_{C}^{[-1]} \circ \pi_{C \wedge D}^{[-1]} & \sim \pi_{C \wedge D}^{[-1]} .
\end{aligned}
$$

Proof. The splitting maps arise from

$$
\phi_{C}: \bar{Y}_{\left.\mathcal{G}\right|_{C}} \times \bar{Y}_{\mathcal{G} / C} \longrightarrow \bar{Y}_{\mathcal{G}}
$$

by choosing a point in $\bar{Y}_{\mathcal{G} / C}$.

Now, the composition law implies that

$$
\pi_{C} \circ \phi_{D}=\phi_{C \wedge D} \circ\left(\pi_{C \wedge D} \times\left(\phi_{f} \circ \pi_{(C+D) / D}\right)\right): \bar{Y}_{\left.\mathcal{G}\right|_{D}} \times \bar{Y}_{\mathcal{G} / D} \longrightarrow \bar{Y}_{\left.\mathcal{G}\right|_{C}},
$$

where $f$ is the natural morphism

$$
f:\left.(\mathcal{G} / D)\right|_{(C+D) / D} \longrightarrow\left(\left.\mathcal{G}\right|_{C}\right) /(C \wedge D) .
$$

If $C=D$, then the right-hand side is just the projection

$$
\bar{Y}_{\left.\mathcal{G}\right|_{C}} \times \bar{Y}_{\mathcal{G} / C} \longrightarrow \bar{Y}_{\left.\mathcal{G}\right|_{C}}
$$

and we thus obtain the desired splitting. More generally, if we choose a point in $\bar{Y}_{\mathcal{G} / D}$, we obtain the second identity. The other two identities follow similarly from the composition law.

REMARK. In particular, the associated retractions satisfy

$$
\left(\pi_{C}^{[-1]} \circ \pi_{C}\right) \circ\left(\pi_{D}^{[-1]} \circ \pi_{D}\right) \sim \pi_{C \wedge D}^{[-1]} \circ \pi_{C \wedge D},
$$

and thus commute up to homotopy. It follows that any associated (co)homology group is graded by $\mathcal{C}_{\mathcal{G}}$; that is, splits as a direct sum indexed by $\mathcal{C}_{\mathcal{G}}$. To be precise, for any $C \in \mathcal{C}_{\mathcal{G}}$, let

$$
H_{*}\left(\bar{Y}_{\mathcal{G}}\right)[C]
$$

denote the subspace consisting of homology classes fixed by $\pi_{C}^{[-1]} \circ \pi_{C}$ and annihilated by $\pi_{D}^{[-1]} \circ \pi_{D}$ for $D \subsetneq C$. (Equivalently, these are the classes in the image of $\pi_{C}^{[-1]}$ which are annihilated by $\pi_{D}$ for $D \subsetneq C$.) Since these are retractions, so idempotents on (co)homology, 
and commute, we find that

$$
H_{*}\left(\bar{Y}_{\mathcal{G}}\right)=\bigoplus_{C \in \mathcal{C}_{\mathcal{G}}} H_{*}\left(\bar{Y}_{\mathcal{G}}\right)[C]
$$

and more generally

$$
\left(\pi_{C}^{[-1]} \circ \pi_{C}\right) H_{*}\left(\bar{Y}_{\mathcal{G}}\right)=\bigoplus_{D \subset C} H_{*}\left(\bar{Y}_{\mathcal{G}}\right)[D]
$$

Proposition 2.9. If $V^{*} \notin \mathcal{G}$, then $\bar{Y}_{\mathcal{G} \cup V^{*}}$ is a projective space bundle over $\bar{Y}_{\mathcal{G}}$.

Proof. Let $G_{1}, \ldots, G_{m}$ be the maximal elements of $\mathcal{G}$; note that by adjoining suitable hyperplanes, we can force $\sum_{i} G_{i}=V^{*}$ without changing $\bar{Y}_{\mathcal{G}}$. We thus need to show that the set of points in $\mathbb{P}(V)$ compatible with a given point in $\bar{Y}_{\mathcal{G}}$ form a projective space. But the compatibility condition is simply that the projection to each $\mathbb{P}_{G_{i}}$, if defined, has the correct value. If we choose representatives $p_{i} \in V / G_{i}^{\perp} \backslash 0$, then a point in $V \backslash 0$ is compatible if and only if it is of the form $\sum_{i} \alpha_{i} p_{i}$; it follows that the preimage is $\mathbb{P}^{m-1}$ as desired.

Proposition 2.10. If $\mathcal{G}=\mathcal{G}^{\prime} \cup\{G\}$, and $G$ is not a maximal element of $\mathcal{G}$, then $\bar{Y}_{\mathcal{G}}$ is the blow-up of $\bar{Y}_{\mathcal{G}^{\prime}}$ along the image $d_{G}^{\prime}$ of the injective map

$$
\phi_{G}: \bar{Y}_{\left.\mathcal{G}^{\prime}\right|_{G}} \times \bar{Y}_{\mathcal{G} / G} \longrightarrow \bar{Y}_{\mathcal{G}^{\prime}} .
$$

The exceptional divisor is the image $d_{G}$ of the injective map

$$
\phi_{G}: \bar{Y}_{\left.\mathcal{G}\right|_{G}} \times \bar{Y}_{\mathcal{G} / G} \longrightarrow \bar{Y}_{\mathcal{G}} \text {. }
$$

Proof. The composition law gives

$$
\phi_{\iota} \circ \phi_{G}=\phi_{G} \circ\left(\phi_{\iota} \times 1\right)
$$

and thus $\phi_{\iota}\left(d_{G}\right)=d_{G}^{\prime}$. Since $\operatorname{dim}\left(d_{G}\right)=\operatorname{dim}\left(\bar{Y}_{\mathcal{G}}\right)-1>\operatorname{dim}\left(d_{G}^{\prime}\right)$ and $d_{G}$ is a projective space bundle over $d_{G}^{\prime}$, it remains only to check that $\phi_{\iota}$ is injective on the complement of $d_{G}$, which is immediate.

REMARK. As in [4] (which used two special cases of this proposition), this immediately gives an inductive proof that $\bar{Y}_{\mathcal{G}}$ is a smooth, irreducible variety, since by the above proposition and Lemma 2.1, it can be obtained from a product of projective spaces by a sequence of blow-ups; it also follows that $\bar{Y}_{\mathcal{G}}(\mathbb{R})$ is a smooth, connected manifold for $\mathcal{G} \in \operatorname{Build}(\mathbb{R})$. Also note the consequence that the normal bundle of $d_{G}^{\prime}$ is trivial if $G$ is minimal in $\mathcal{G}$, since then $d_{G}$ is a product bundle.

Note that this construction of $\bar{Y}_{\mathcal{G}}$ as an iterated blow-up can almost certainly be generalized. Indeed, Keel's construction [13] of $\bar{M}_{0, n+1}$ as a blow-up of $\left(\mathbb{P}^{1}\right)^{n-1}$ is not of the above form.

In general, for any category $\mathbf{C}$, we define a universal operad in $\mathbf{C}$ to be a functor $F:$ Build $\rightarrow$ $\mathbf{C}$ that also associates a morphism

$$
\phi_{f}: F\left(\left.\mathcal{G}^{\prime}\right|_{\operatorname{ker}\left(f^{*}\right)} \oplus \mathcal{G}\right) \longrightarrow F\left(\mathcal{G}^{\prime}\right)
$$

to every weak morphism, satisfying the composition law

$$
\phi_{g} \circ \phi_{1 \oplus f}=\phi_{g \circ f} \circ \phi_{\left.g\right|_{\mathrm{im}(f)} \oplus 1} .
$$


A natural transformation between universal operads will be said to be operadic if it is compatible with the composition law in the obvious way. A universal cooperad in $\mathbf{C}$ is simply a universal operad in $\mathbf{C}^{\text {op }}$.

REMARK. A universal operad in a tensor category, equipped with natural isomorphisms $F\left(\mathcal{G} \oplus \mathcal{G}^{\prime}\right) \rightarrow F(\mathcal{G}) \otimes F\left(\mathcal{G}^{\prime}\right)$ (compatible with symmetry and associativity), induces (by restriction to the braid arrangements) an operad in the usual sense; this is not true for a general universal operad, however. Also, given a topological universal operad, we may take the homology of $F$ (with appropriate coefficients), and thus obtain a universal operad in the appropriate category of modules (or, taking cohomology, a universal cooperad of rings, assuming the topological universal operad respects products).

\section{Poset homology and operad maps}

In this section, and until further notice, we restrict our attention to the case $\mathcal{G} \in \operatorname{Build}(\mathbb{R})$. In this case, there is a significant difference between odd- and even-dimensional elements of $\mathcal{G}$ : for $\operatorname{dim}(G)>1, \mathbb{P}_{G}(\mathbb{R})$ is orientable if and only if $\operatorname{dim}(G)$ is even. This suggests that evendimensional elements will have particular significance in the homology of $\bar{Y}_{\mathcal{G}}(\mathbb{R})$.

More generally, let $\Pi_{\mathcal{G}}^{(m)}$ be the subposet of $\mathcal{C}_{\mathcal{G}}$ consisting of elements $A$ that can be written as direct sums of elements $G \in \mathcal{G}$ with $\operatorname{dim}(G)$ a multiple of $m$. (In the case of the braid arrangement $\mathcal{G}=\overline{A_{n-1}}, \Pi_{\mathcal{G}}^{(m)}$ is the poset of partitions of $\{1,2, \ldots, n\}$ into parts all of size congruent to 1 modulo $m$.) For the remainder of this section, we fix a choice of $m$; after this section, we will in fact take $m=2$ almost exclusively.

For any element $A \in \Pi_{\mathcal{G}}^{(m)}$ and any commutative ring $R$, we define $H_{*}([0, A] ; R)$ to be the homology of the chain complex $C_{*}([0, A] ; R)$ in which $C_{k+1}([0, A] ; R)$ is the free $R$-module spanned by chains

$$
\left(0<A_{1}<\ldots<A_{k}<A\right)
$$

in $\Pi_{\mathcal{G}}^{(m)}$, and the boundary map is defined by

$$
\partial\left(0<A_{1}<\ldots<A_{k}<A\right)=\sum_{i}(-1)^{i}\left(0<A_{1}<\ldots<\hat{A}_{i}<\ldots<A_{k}<A\right) .
$$

(Aside from a shift in degree, this is the reduced homology of the order complex of the open interval $(0, A)$.) By convention, $C_{*}([0,0] ; R)=0$ except in degree 0 , where it is spanned by the single chain $(0)$.

It will be convenient to consider an alternate chain complex giving the same homology, defined in terms of ' $\mathcal{G}$-forests' (these are called 'nested sets' in [4], but we feel 'forest' is more evocative of the relevant combinatorics, especially in the braid case).

Definition 1. A $\mathcal{G}$-forest is a subset $F \subset \mathcal{G}$ such that every collection of pairwise incomparable elements of $F$ forms a decomposition; the root of $F$ is the space

$$
\operatorname{root}(F):=\sum_{G \in F} G \in \mathcal{C}_{\mathcal{G}} .
$$

(Note that this subspace will not be an element of $F$ in general, but merely a direct sum of elements of $F$.) Given $G \in F$, the child of $G$ in $F$ is the space

$$
\operatorname{child}_{F}(G):=\sum_{\substack{H \in F \\ H \subsetneq G}} H .
$$

A forest $F$ is said to be $m$-divisible if every element of $F$ has dimension a multiple of $m$. 
REMARK. There is a natural bijection between $\overline{A_{n-1}}$-forests and forests in the usual sense in which the leaves are labeled $1, \ldots, n$. Given such a forest, if one labels each internal node by the set of its descendant leaves, the resulting collection of subsets forms an $\overline{A_{n-1}}$-forest.

The name 'forest' is justified by the following lemma.

Lemma 3.1. Let $F$ be a $\mathcal{G}$-forest. For any element $G \in F$, the set of elements of $F$ containing $G$ forms a chain.

Proof. Indeed, if $H_{1}, H_{2} \in F$ are incomparable elements, then by definition they form a decomposition; in particular, $G$ cannot be contained in both.

In other words, $F$ has a natural structure of a forest with nodes labeled by elements of $\mathcal{G}$, compatible with inclusion; the root in our sense is simply the direct sum of the labels of the roots.

Now, for $A \in \Pi_{\mathcal{G}}^{(m)}$, define a chain complex $C_{*}^{f}(A)$ as follows. The $R$-module $C_{k}^{f}(A)$ is spanned by ordered $m$-divisible forests $F$ with root $A$ and $k$ nodes, but with different orderings identified, up to the obvious sign factor. For $G \in \mathcal{G}$, we define

$$
\partial_{G} F= \begin{cases}(-1)^{i-1} F \backslash G, & G=F_{i}, \\ 0, & G \notin F ;\end{cases}
$$

the boundary map is then given by the sum of $\partial_{G}$ with $G$ ranging over proper subspaces of components of $A$. We will also need a concatenation operation

$$
G \cdot\left(F_{1}, F_{2}, \ldots, F_{n}\right)=\left(G, F_{1}, F_{2}, \ldots, F_{n}\right),
$$

or 0 if the result is not a forest.

The following proof is adapted from [17, Section 2.6], which essentially considered the case $\Pi_{A_{n}}^{(1)}$ with $A=V$.

Theorem 3.2. For all $A \in \Pi_{\mathcal{G}}^{(m)}$, there is a canonical isomorphism

$$
H_{*}([0, A]) \cong H_{*}^{f}(A) .
$$

Proof. If $A=0$, then the result is immediate (in both cases, $H_{0}=\mathbb{Z}$ and all other homology groups are trivial); we may thus assume $A \neq 0$, and thus both complexes are trivial in degree 0 .

Now, given a chain

$$
0<A_{1}<A_{2}<\ldots<A_{k}<A,
$$

consider the (partially closed) simplex of numbers $0 \leqslant \tau_{k}<\ldots<\tau_{1}<\tau_{0}=1$. The remainder of the closure of this simplex is naturally identified with the disjoint union of simplices corresponding to chains with steps removed (if $\tau_{k}=\tau_{k-1}$, then remove $A_{k}$ ); as a result, we can glue together all of the simplices to obtain a geometric simplicial complex $\Sigma$. The result is simply the order complex of $(0, A]$, so its local homology at the point $(0<A)$ is

$$
H_{*}(\Sigma, \Sigma \backslash\{(0<A)\})=H_{*+1}([0, A]) .
$$

Similarly, given a forest $F$, consider labelings $\tau^{\prime}$ of the nodes, subject to the condition that the labels sum to 1, are nonnegative, and the labels of all nonroots are positive. Again, if we include labelings of subforests (with the same root), with the convention that $\tau^{\prime}=0$ for removed nodes, we obtain a closed simplex, and a geometric simplicial complex $\Sigma^{f}$. If $x$ is the 
centroid of the simplex corresponding to the forest $A$, the local homology at $x$ is

$$
H_{*}\left(\Sigma^{f}, \Sigma^{f} \backslash\{x\}\right)=H_{*+1}^{f}(A) .
$$

(Taking local homology with respect to $x$ is equivalent to restricting the chain complex to those simplices containing the open simplex that contains $x$.)

The theorem will follow if we can establish a pointed homeomorphism $\Sigma \cong \Sigma^{f}$. Define a (discontinuous) function $\rho:(0,1) \rightarrow \Pi_{\mathcal{G}}^{(m)}$ by $\rho(t)=A_{l}$ whenever $\tau_{l}<t<\tau_{l-1}$ (with the convention $A_{k+1}=A, \tau_{k+1}=0$ ). Now, if $F$ is the forest consisting of all components of the $A_{k}$, we initially label a node $G$ by the difference between the limsup and the liminf of those $t$ for which $G$ is a component of $\rho(t)$. The only way such a label can be 0 is if $\tau_{0}=0$ and $G$ is a component of $A$ but not $A_{1}$; in particular nonroot nodes have a nonzero label. Since the sum of the labels is uniformly bounded away from 0 and $\infty$, we may rescale to make the sum 1 , and obtain the desired labeling $\tau^{\prime}$. To invert, we rescale so that the highest-weight path from the root has weight 1 , and can then immediately recover the labeled chain.

If we begin with the chain $(0<A)$, we find $\rho \equiv A$. The corresponding forest is just the set of components of $A$, each labeled (after normalization) by $1 / l$ (if $A$ has $l$ components). But this is indeed the centroid of the simplex corresponding to that forest.

REMARK. The general case $m=1$ was established by different means in [8]. Similarly, the case $m>1, \mathcal{G}=\overline{A_{n-1}}$ was established in $[\mathbf{6}]$.

Under this isomorphism, each simplex of $\Sigma^{f}$ is identified with a union of simplices of $\Sigma$, and thus the above isomorphism induces a chain map.

Corollary 3.3. Define a map $\sigma: C_{*}^{f}(A) \rightarrow C_{*}([0, A])$ by

$$
\sigma(F):=\sum_{\pi} \sigma(\pi)\left(0<F_{\pi(1)}<F_{\pi(1)}+F_{\pi(2)}<\ldots<F_{\pi(1)}+\ldots+F_{\pi(k-1)}<A\right),
$$

where the sum is over permutations of the nodes such that if $F_{\pi(i)} \subset F_{\pi(j)}$, then $i \leqslant j$. Then $\sigma$ is a chain map inducing an isomorphism on homology.

A chain of the form

$$
\left(0<F_{\pi(1)}<F_{\pi(1)}+F_{\pi(2)}<\ldots<F_{\pi(1)}+\ldots+F_{\pi(k-1)}<A\right)
$$

will be called a forest chain.

Lemma 3.4. A linear combination of forest chains in $C_{*}([0, A])$ is in the image of $\sigma$ if and only if its boundary is also a linear combination of forest chains.

Proof. This follows from geometric considerations, but can also be shown directly as follows. Suppose we order $F$ in such a way that $F_{i} \subset F_{j}$ implies $i \leqslant j$, and suppose $F_{l}, F_{l+1}$ are incomparable. Then the nonforest chain

$$
\left(0<F_{1}<\ldots<F_{1}+\ldots+F_{l-1}<F_{1}+\ldots+F_{l+1}<\ldots<A\right)
$$

occurs in the differential of precisely two forest chains, namely

$$
\left(0<F_{1}<\ldots<F_{1}+\ldots+F_{l-1}<F_{1}+\ldots+F_{l-1}+F_{l}<F_{1}+\ldots+F_{l+1}<\ldots<A\right)
$$

and

$$
\left(0<F_{1}<\ldots<F_{1}+\ldots+F_{l-1}<F_{1}+\ldots+F_{l-1}+F_{l+1}<F_{1}+\ldots+F_{l+1}<\ldots<A\right) .
$$


Thus the coefficient of this nonforest chain in the differential is 0 if and only if the coefficients of the forest chains are negatives of each other.

Since any two such orderings of $F$ can be connected by a sequence of such transpositions, the claim follows.

REMARK. In particular, a chain map between two complexes $C_{*}([0, A])$ that takes forest chains to forest chains pulls back to a chain map on the associated forest complexes.

Now, define the Whitney homology

$$
W_{*}^{(m)}(\mathcal{G})=\bigoplus_{A \in \Pi_{\mathcal{G}}^{(m)}} H_{*}([0, A]),
$$

a module graded by both the degree in homology and by the poset $\Pi_{\mathcal{G}}^{(m)}$. This, of course, can be computed as the homology of the corresponding sum of poset or forest complexes; thus define, for instance, $C_{*}^{W}(\mathcal{G})=\bigoplus_{A \in \mathcal{C}_{\mathcal{G}}} C_{*}^{W}([0, A])$, and similarly for forests.

Theorem 3.5. The Whitney homology extends to a cooperad such that the maps $\phi_{f}^{*}$ are homogeneous and respect the poset grading:

$$
\phi_{f}^{*}\left(W^{(m)}\left(\mathcal{G}^{\prime}\right)[A]\right)=W^{(m)}\left(\left.\mathcal{G}^{\prime}\right|_{\operatorname{ker}\left(f^{*}\right)} \oplus \mathcal{G}\right)\left[\left(A \cap \operatorname{ker} f^{*}\right) \oplus f^{*}(A)\right] ;
$$

in particular $\phi_{f}^{*}$ vanishes unless

$$
\left(A \cap \operatorname{ker} f^{*}\right) \oplus f^{*}(A) \subset \Pi_{\left.\mathcal{G}^{\prime}\right|_{\operatorname{ker}\left(f^{*}\right)} ^{(m)} \oplus \mathcal{G}} .
$$

Proof. Let $f: \mathcal{G} \rightarrow \mathcal{G}^{\prime}$ be a weak morphism. We define a chain map

$$
\phi_{f}^{*}: C_{*}^{W}\left(\mathcal{G}^{\prime}\right) \longrightarrow C_{*}^{W}\left(\left.\mathcal{G}^{\prime}\right|_{\operatorname{ker}\left(f^{*}\right)}\right) \otimes C_{*}^{W}(\mathcal{G})
$$

on chains $0<\ldots<A_{i}<\ldots<A_{k}=A$ in $\Pi_{\mathcal{G}}^{(m)}$ as follows. If $A \cap \operatorname{ker} f^{*}$ is not in the chain, we set $\phi_{f}^{*}=0$. Otherwise, let $l$ be the index of $A \cap \operatorname{ker} f^{*}$ in the chain, and define

$$
\begin{aligned}
\phi_{f}^{*}\left(0<\ldots<A_{i}<\ldots<A\right)= & \left(0<\ldots<A_{i} \cap \operatorname{ker} f^{*}<\ldots<A_{l} \cap \operatorname{ker} f^{*}=A \cap \operatorname{ker} f^{*}\right) \\
& \otimes\left(0=f^{*}\left(A_{l}\right)<\ldots<f^{*}\left(A_{l+i}\right)<\ldots<f^{*}(A)\right) \\
& \in C_{l}^{W}\left(\left.\mathcal{G}^{\prime}\right|_{\operatorname{ker}\left(f^{*}\right)}\right) \otimes C_{k-l}^{W}(\mathcal{G}) .
\end{aligned}
$$

Since deleting a step cannot introduce $A \cap \operatorname{ker} f^{*}$ to the chain, the differential cannot leave the set of 'bad' chains (that is, in the kernel of $\phi_{f}^{*}$ ). And the only way to produce a bad chain by deleting a node is to delete $A \cap \operatorname{ker} f^{*}$; but the corresponding term is missing from the differential on the image space. Therefore $\phi_{f}^{*}$ is indeed a chain map. Moreover, it is easily seen to satisfy the composition law. Finally, we can extend it to a chain map

$$
\phi_{f}^{*}: C_{*}^{W}\left(\mathcal{G}^{\prime}\right) \longrightarrow C_{*}^{W}\left(\left.\mathcal{G}^{\prime}\right|_{\operatorname{ker}\left(f^{*}\right)} \oplus \mathcal{G}\right)
$$

by composing with the shuffle product

$$
C_{*}^{W}\left(\left.\mathcal{G}^{\prime}\right|_{\operatorname{ker}\left(f^{*}\right)}\right) \otimes C_{*}^{W}(\mathcal{G}) \longrightarrow C_{*}^{W}\left(\left.\mathcal{G}^{\prime}\right|_{\operatorname{ker}\left(f^{*}\right)} \oplus \mathcal{G}\right),
$$

obtaining the map on homology as required.

REMARK 1. Since the Whitney homology is a direct sum, it is equivalent to consider only the induced maps

$$
\phi_{f}^{*}: H_{*}\left([0, A]_{\mathcal{G}^{\prime}}\right) \longrightarrow H_{*}\left(\left.\left[0, A \cap \operatorname{ker} f^{*} \oplus f^{*}(A)\right]_{\mathcal{G}^{\prime}}\right|_{\operatorname{ker}\left(f^{*}\right)} \oplus \mathcal{G}\right)
$$


where $A \cap \operatorname{ker} f^{*} \oplus f^{*}(A) \in \Pi_{\left.\mathcal{G}^{\prime}\right|_{\text {ker } f^{*}} \oplus \mathcal{G}}^{(m)}$, and similarly for the chain maps.

REMARK 2. The case of the diagonal morphism $\Delta: \mathcal{G} \rightarrow \mathcal{G} \oplus \mathcal{G}$ is of particular interest:

$$
\phi_{\Delta}^{*}\left(0<\ldots<\left(A_{i}, B_{i}\right)<\ldots<(A, B)\right)= \begin{cases}0<\ldots<A_{i}+B_{i}<\ldots A+B & \text { if } A \cap B=0, \\ 0 & \text { otherwise. }\end{cases}
$$

Composing with the shuffle product induces a ring structure on the Whitney homology, graded by $\Pi_{\mathcal{G}}^{(m)}$.

Since $\phi_{f}^{*}$ takes forest chains to forest chains, we conclude the following.

Corollary 3.6. For each weak morphism $f: \mathcal{G} \rightarrow \mathcal{G}^{\prime}$, there is a chain map

$$
\phi_{f}^{*}: C_{*}^{W f}\left(\mathcal{G}^{\prime}\right) \longrightarrow C_{*}^{W f}\left(\left.\mathcal{G}^{\prime}\right|_{\operatorname{ker}\left(f^{*}\right)} \oplus \mathcal{G}\right)
$$

producing a commutative diagram

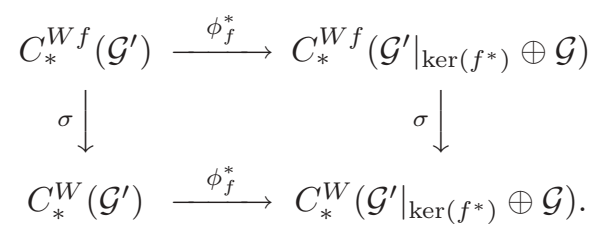

Thus, in addition to the cooperad structure on the Whitney homology itself, we also obtain two cooperads of chain complexes, and an operadic homotopy between them. It is unclear, however, how to define the cooperad structure on the forest complex without passing through the poset complex.

It turns out that the relation to De Concini-Procesi models is more convenient in terms of poset and forest cohomology. Of course, the cooperads of homology chain complexes immediately dualize to operads on the cohomology chain complexes, and thus give rise to an operad on the Whitney cohomology (defined in the obvious way).

Even this is not quite the right structure, however. Note that the induced chain maps

$$
\left(\phi_{f}\right)_{*}: C^{*}\left(\left.[0, B \oplus C]_{\mathcal{G}^{\prime}}\right|_{\operatorname{ker}\left(f^{*}\right)} \oplus \mathcal{G}\right) \longrightarrow C^{*}\left([0, A]_{\mathcal{G}^{\prime}}\right)
$$

are 0 unless $B=A \cap \operatorname{ker} f^{*}, C=f^{*}(A)$; in other words, we must have a short exact sequence

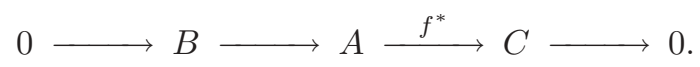

In particular, $\operatorname{dim}(A)=\operatorname{dim}(B)+\operatorname{dim}(C)$, and we may thus shift the degrees of the complex by this dimension without affecting homogeneity of $\left(\phi_{f}\right)^{*}$. To be precise, we will use the chain complex

$$
C^{\operatorname{dim} A-*}\left([0, A]_{\mathcal{G}^{\prime}}\right),
$$

which becomes a homology complex since the differential now decreases the degree.

A more subtle correction is a certain twisting of the operad structure. For any real vector space $V$, let $\operatorname{or}(V):=H_{\operatorname{dim}\left(V^{*}\right)}\left(V^{*}, V^{*} \backslash\{0\}\right)$ be the corresponding orientation module; note in particular the canonical isomorphisms

$$
\operatorname{or}(V) \cong \tilde{H}_{\operatorname{dim}(V)-1}\left(S\left(V^{*}\right)\right),
$$

and when $\operatorname{dim}(V)$ is even,

$$
\operatorname{or}(V) \cong \tilde{H}_{\operatorname{dim}(V)-1}\left(\mathbb{P}\left(V^{*}\right)\right)
$$


In any event, every short exact sequence $0 \rightarrow V \rightarrow W \rightarrow X \rightarrow 0$ induces a canonical isomorphism $\operatorname{or}(V) \otimes \operatorname{or}(X) \rightarrow \operatorname{or}(W)$. Thus every nonzero $\phi_{f}^{*}$ induces an isomorphism $\operatorname{or}(A) \cong$ or $(B \oplus C)$, and we obtain an operad map

$$
\left(\phi_{f}\right)_{*}: C^{\operatorname{dim}(B \oplus C)-*}\left(\left.[0, B \oplus C]_{\mathcal{G}^{\prime}}\right|_{\operatorname{ker}\left(f^{*}\right)} \oplus \mathcal{G}\right) \otimes \operatorname{or}(B \oplus C) \longrightarrow C^{\operatorname{dim}(A)-*}\left([0, A]_{\mathcal{G}^{\prime}}\right) \otimes \operatorname{or}(A),
$$

as well as associated maps on (degree-reversed) cohomology.

We may now state our main theorem. Recall that the notation $[A]$ refers to the $A$-graded piece of the homology; see the remark following Corollary 2.8. Also, we identify the real algebraic variety $\bar{Y}_{\mathcal{G}}$ with its real locus $\bar{Y}_{\mathcal{G}}(\mathbb{R})$, whenever this can be done without causing confusion.

Theorem 3.7. Let $\mathcal{G}$ be a real building set, and $A \in \Pi_{\mathcal{G}}$. If $A \notin \Pi_{\mathcal{G}}^{(2)}$, then

$$
2 H_{*}\left(\bar{Y}_{\mathcal{G}}\right)[A]=0 ;
$$

otherwise, there is a natural, operadic, isomorphism

$$
H_{(2)}^{\operatorname{dim}(A)-*}\left([0, A]_{\mathcal{G}}\right) \otimes \operatorname{or}(A) \longrightarrow 2 H_{*}\left(\bar{Y}_{\mathcal{G}}\right)[A] .
$$

REMARK 1. A similar result (minus the operad structure, and requiring some additional hypotheses for naturality) was already known $[\mathbf{1 0}]$ (see also $[\mathbf{5}, \mathbf{1 6}, \mathbf{1 9}]$ for the ring structure of cohomology) for the complement of a real subspace arrangement, namely an isomorphism of its homology with

$$
\bigoplus_{A \in \Pi_{\mathcal{G}}^{(1)}} H_{(1)}^{\operatorname{dim}(A)-*}\left([0, A]_{\mathcal{G}}\right) \otimes \operatorname{or}(A) .
$$

One curious consequence of this similarity is that if $\mathcal{G}$ is obtained from a complex building set by viewing each space as a real space of twice the dimension, then (since every subspace now has even dimension) there is an isomorphism

$$
2 H_{*}\left(\bar{Y}_{\mathcal{G}}\right) \cong H_{*}\left(V^{*}-\mathcal{G}\right) .
$$

In particular, the homology of $\bar{Y}_{\mathcal{G}}(\mathbb{R})$ can be arbitrarily complicated (even for hyperplane arrangements, by the remark following Lemma 2.2), since the same holds for the complements of complex subspace arrangements. Indeed, any finite simplicial complex is homeomorphic to the order complex of a finite atomic lattice (its lattice of faces), and thus, reversing inequalities, to the order complex of a finite coatomic lattice. But any finite coatomic lattice can be represented as a lattice of subspaces of the space of complex-valued functions on the coatoms (each element corresponds to the space of functions vanishing on the coatoms bounding it).

REMARK 2. Dually, there is a natural isomorphism from the cohomology (modulo 2-torsion) of $\bar{Y}_{\mathcal{G}}(\mathbb{R})$ to the (suitably twisted) Whitney homology of $\Pi_{\mathcal{G}}^{(2)}$, and operadicity (applied to the diagonal map) implies that this is an isomorphism of (poset-graded) rings.

The main theorem has an important consequence for the moduli space of stable genus 0 curves.

Corollary $3.8\left[\mathbf{7}\right.$, Conjecture 2.13]. The groups $2 H_{*}\left(\overline{M_{0, n}}(\mathbb{R}) ; \mathbb{Z}\right)$ and $2 H^{*}\left(\overline{M_{0, n}}(\mathbb{R}) ; \mathbb{Z}\right)$ are free.

Proof. As remarked above, we have an isomorphism $\overline{M_{0, n+1}} \cong \bar{Y}_{\overline{A_{n-1}}}$ of algebraic varieties, and thus of their respective real loci. It follows that $2 H_{*}\left(\overline{M_{0, n}}(\mathbb{R}), \mathbb{Z}\right)$ is isomorphic to a direct 
sum of cohomology groups of subposets of the poset of set partitions with all parts odd. But this poset is Cohen-Macaulay, and thus its cohomology is free (and supported in the appropriate degree). The remaining claim follows from the universal coefficient theorem.

REMARK 1. The same argument applies to an arbitrary Coxeter arrangement (see [11]) again because the relevant posets are Cohen-Macaulay.

REMARK 2. Note, however, that the explicit presentation of the ring

$$
H^{*}\left(\overline{M_{0, n}}(\mathbb{R}) ; \mathbb{Z}\right) / H^{*}\left(\overline{M_{0, n}}(\mathbb{R}) ; \mathbb{Z}\right)[2]
$$

given in [7] does not follow from the present methods. It is, of course, far too much to hope for such a presentation for completely general building sets (or even for general hyperplane arrangements, by the discussion following Lemma 2.2); for instance, the cohomology ring need not be generated in degree 1 in that case.

\section{Blow-ups and homology}

The construction of $\bar{Y}_{\mathcal{G}}$ via repeated blow-ups turns out to have extremely useful consequences in homology. As we are interested in the topological consequences of this, it will be useful to have a more topological (or, more precisely, differentiable rather than algebraic) version of blowing-up. In addition to real and complex blow-ups, corresponding to algebraic blowing-up of real or complex varieties, there is a third 'spherical' blow-up that it will be useful to consider. (For an early application of such blow-ups to subspace arrangements, see [2, §5].) In fact, the spherical blow-up is in some sense universal; the other blow-ups can be constructed as quotients of the spherical blow-up.

The basic idea of the spherical blow-up is to replace a submanifold $Y$ by the sphere bundle $N_{Y}(X) / \mathbb{R}^{+}$. That is, the blow-up is a new space $\tilde{X}$ equipped with a continuous (smooth) map to $X$ which is an isomorphism outside $Y$, and such that the preimage of a given point in $Y$ is identified with the space of unit normal vectors to $Y$ at that point. The result is homotopic to the complement of $Y$ in $X$, but has the merit of being compact and almost smooth; the failure to be smooth being that if $X$ is a compact manifold, then $\tilde{X}$ is a compact manifold with boundary (the preimage of $Y$ ). Similarly, if $X$ is a manifold with boundary, then $\tilde{X}$ can have corners. Recall that a smooth manifold with corners is a (paracompact, Hausdorff) space $X$ with a covering $U_{i}$ by open sets, each homeomorphic to a space $\mathbb{R}^{p_{i}} \times\left(\mathbb{R}^{\geqslant 0}\right)^{q_{i}}$, and in such a way that the compatibility maps are $C^{\infty}$. We extend this to pairs $(X, Y)$ by insisting that each $Y \cap U_{i}$ either be empty or an intersection of coordinate hyperplanes. (This is essentially just a condition that $Y$ meets the boundary and corners of $X$ transversely.) In particular, given a pair, we may associate a normal bundle $N_{Y}(X)$, which on a patch $U_{i} \cap Y$ is the quotient of the cone $\mathbb{R}^{p_{i}} \times\left(\mathbb{R}^{\geqslant 0}\right)^{q_{i}}$ by the subspace $U_{i} \cap Y$.

The spherical blow-up of the pair $(X, Y)$ is then defined as follows. If $Y$ is empty, then the blow-up of $(X, Y)$ is itself; if $X$ is a cone bundle over $Y$, then the blow-up is the pair $(\tilde{X}, \tilde{Y})$, where $\tilde{X}$ is the closure of the subset

$$
X-Y \subset X \times X / \mathbb{R}^{+},
$$

and $\tilde{Y} \cong X / \mathbb{R}^{+}$is the preimage of $Y$ in $\tilde{X}$. In general, any pair $(X, Y)$ looks locally like one of the above two examples, and the above constructions are sufficiently compatible to give a global construction. In particular, note that $(\tilde{X}, \tilde{Y})$ is a smooth pair with corners, and the induced map $\tilde{X}-\tilde{Y} \rightarrow X-Y$ is a diffeomorphism. 
For instance, if $X \subset \mathbb{R}^{n}$ and $Y \subset X$ consists of a single point, then there is a natural projection $X \backslash Y \rightarrow S^{n-1}$, and $\tilde{X}$ is the closure of $X \backslash Y$ in $\mathbb{R}^{n} \times S^{n-1}$. The subset $\tilde{Y}$ can then be viewed as the set of all unit vectors pointing toward $X$ from $Y$.

Since $\tilde{Y} \cong N_{Y}(X) / \mathbb{R}^{+}$, one can define other blow-ups as push-forwards of appropriate surjections from $\tilde{Y}$. In particular, the real blow-up corresponds to the map $N_{Y}(X) / \mathbb{R}^{+} \rightarrow$ $N_{Y}(X) / \mathbb{R}^{*}$ (valid so long as $N_{Y}(X)$ is a vector bundle, for example, if $Y$ is disjoint from the corners of $X$ ), while the complex blow-up corresponds to a map $N_{Y}(X) / \mathbb{R}^{+} \rightarrow N_{Y}(X) / \mathbb{C}^{*}$, assuming such a complex structure exists. Both of these, unlike the spherical blow-up, preserve smoothness; however, the spherical blow-up is a useful technical tool, both because it maps to these cases and because it has the homotopy type of $X \backslash Y$. (See also [9], which considers the analog of $\bar{Y}_{\mathcal{G}}(\mathbb{R})$ replacing real blow-ups with spherical blow-ups.)

It turns out that in general, there is a long exact sequence relating the homology of $X$, the homology of the blow-up, and the homology of the mapping cone of the projection $\pi$ : $\pi^{-1}(Y) \rightarrow Y$. For a continuous map $f: X \rightarrow Y$, recall that the mapping cone $M_{f}$ is defined as the quotient of cone $(X) \cup Y$ by identifying each point in $X$ with its image in $Y$. (By convention, if $X=\emptyset$, then cone $(X)$ consists of a single point.) Note that commutative diagrams of the form

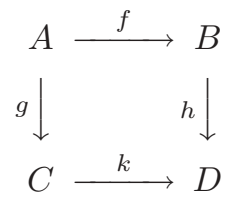

induce continuous maps between mapping cones; moreover, there is a natural homeomorphism

$$
M_{(g, h): M_{f} \longrightarrow M_{k}} \cong M_{(f, k): M_{g} \longrightarrow M_{h}} .
$$

Given a map of pairs $f:(A, B) \rightarrow(C, D)$, define $H_{*}(f):=H_{*}\left(M_{f: A \rightarrow C}, M_{f: B \rightarrow D}\right)$. Note that if $B=D=\emptyset$, then this is the reduced homology of $M_{f: A \rightarrow C}$; if $f$ is also an inclusion map, then $H_{*}(f) \cong H_{*}(C, A)$.

Lemma 4.1. Let $f:(A, B) \rightarrow(C, D)$ be a continuous map of pairs. Then there is a long exact sequence

$$
\cdots \longrightarrow H_{*}(A, B) \stackrel{f_{*}}{\longrightarrow} H_{*}(C, D) \longrightarrow H_{*}(f) \longrightarrow H_{*-1}(A, B) \longrightarrow \cdots
$$

functorial in the sense that, for any commutative square

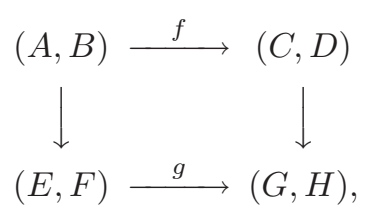

the corresponding diagram

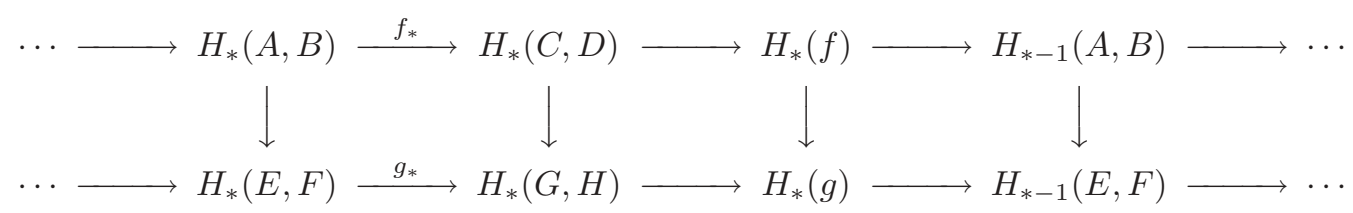

commutes. 
Proof. If $B=D=\emptyset, A, C \neq \emptyset$, then we have the commutative diagram

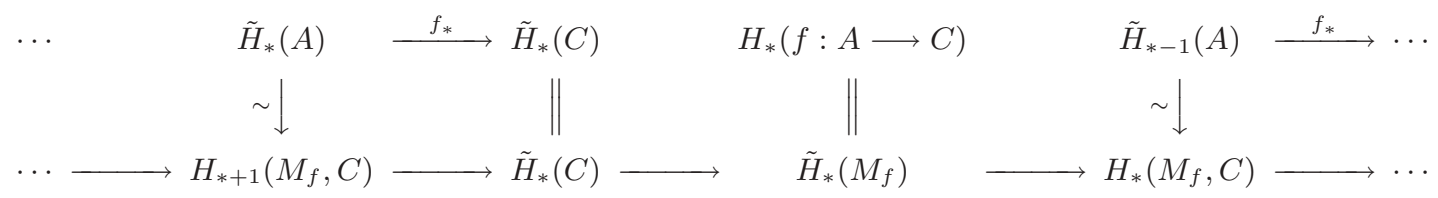

which gives rise to a reduced homology version of the desired sequence. For the general case, let $M_{A, B}$ denote the mapping cone of the inclusion $B \rightarrow A$, and observe that the mapping cone of

$$
f: M_{A, B} \longrightarrow M_{C, D}
$$

is homeomorphic to the mapping cone of the inclusion

$$
M_{f: B \rightarrow D} \longrightarrow M_{f: A \rightarrow C} .
$$

Thus we have the commutative diagram

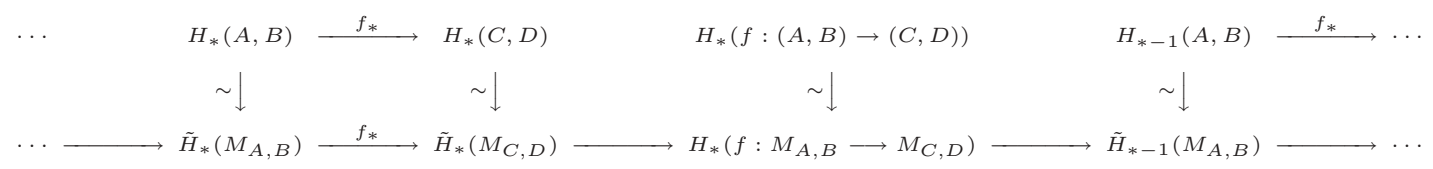

Remark. More generally, given two maps $f:(A, B) \rightarrow(C, D), g:(C, D) \rightarrow(E, F)$, there is a long exact sequence

$$
\cdots \longrightarrow H_{*}(f) \stackrel{(1, g)}{\longrightarrow} H_{*}(g \circ f) \stackrel{(f, 1)}{\longrightarrow} H_{*}(g) \longrightarrow H_{*-1}(f) \longrightarrow \cdots
$$

which gives the above result when $(A, B)=(\emptyset, \emptyset)$, and when $B=D=E=F=\emptyset$ gives the reduced homology version.

Corollary 4.2. Suppose the map $f:(A, B) \rightarrow(C, D)$ induces an isomorphism on relative homology. Then there is a functorial long exact sequence

$$
\cdots \longrightarrow H_{*}(A) \stackrel{f_{*}}{\longrightarrow} H_{*}(C) \longrightarrow H_{*}(f: B \longrightarrow D) \longrightarrow H_{*-1}(A) \longrightarrow \cdots
$$

Proof. It suffices to show that the inclusion maps induce an isomorphism

$$
H_{*}(f: B \longrightarrow D) \cong H_{*}(f: A \longrightarrow C) .
$$

From the long exact sequence of relative homology, this is equivalent to the vanishing of the homology group

$$
H_{*}(f:(A, B) \longrightarrow(C, D))
$$

which by the mapping cone long exact sequence is equivalent to the claim that $f_{*}: H_{*}(A, B) \rightarrow$ $H_{*}(C, D)$ is an isomorphism.

In the case of a blow-up, we have the following.

TheOREM 4.3. Let $(X, Y)$ be a smooth manifold pair with corners, and let $(\tilde{X}, \tilde{Y})$ be a corresponding (spherical, real, complex) blow-up. Then there is a long exact sequence

$$
\cdots \longrightarrow H_{*}(\tilde{X}) \longrightarrow H_{*}(X) \longrightarrow H_{*}(\tilde{Y} \longrightarrow Y) \longrightarrow H_{*-1}(\tilde{X}) \cdots
$$

Moreover, the map $H_{*}(X) \rightarrow H_{*}(\tilde{Y} \rightarrow Y)$ factors through the corresponding map $H_{*}(X) \rightarrow$ $H_{*}\left(\left(N_{Y}(X) / \mathbb{R}^{+}\right) \rightarrow Y\right)$. 
Proof. It suffices to show that the natural map $\pi_{*}: H_{*}(\tilde{X}, \tilde{Y}) \rightarrow H_{*}(X, Y)$ is an isomorphism; indeed, then the long exact sequence follows from Corollary 4.2, and the fact that the map factors follows from functoriality.

Now, if $U$ is an open subset of a patch, then $\pi_{*}: H_{*}\left(\pi^{-1}(U), \pi^{-1}(U \cap Y)\right) \rightarrow H_{*}(U, U \cap Y)$ is an isomorphism, since $\pi^{-1}(U \cap Y)$ and $U \cap Y$ are both deformation retracts of neighborhoods. It then follows from Mayer-Vietoris that this holds whenever $U$ is a finite union of such subsets, and then by taking a direct limit, that it holds in general.

The fact that the map factors through the spherical case is surprisingly powerful, and in particular gives short exact sequences in many cases.

Corollary 4.4. Let $(X, Y)$ and $(\tilde{X}, \tilde{Y})$ be as above. In the case of a complex blow-up, the connecting map $H_{*}(X ; R) \rightarrow H_{*}(\tilde{Y} \rightarrow Y ; R)$ is always 0 . In the case of a real blow-up, the connecting map is 0 if either $R$ has characteristic 2 or each component of $Y$ has odd codimension, and in general factors through multiplication by 2 .

Proof. Indeed, it suffices to consider the case of $\mathbb{C}^{p}$ or $\mathbb{R}^{p}$ blown up at the origin, for which the claims are immediate.

We now consider the implications of the blow-up long exact sequence for real De ConciniProcesi models.

Corollary 4.5. Suppose $G \in \mathcal{G}$, that $\mathcal{G}^{\prime}:=\mathcal{G} \backslash\{G\}$ is a building set, and that $G \notin \mathcal{C}_{\mathcal{G}^{\prime}}$; let

$$
d_{G}=\bar{Y}_{\left.\mathcal{G}\right|_{G} \oplus \mathcal{G} / G}
$$

be the exceptional divisor, and

$$
d_{G}^{\prime}=\bar{Y}_{\left.\mathcal{G}^{\prime}\right|_{G} \oplus \mathcal{G} / G}
$$

its image. The homology groups of $\bar{Y}_{\mathcal{G}}(\mathbb{R})$ and $\bar{Y}_{\mathcal{G}^{\prime}}(\mathbb{R})$ are related as follows. If $G \not \subset A$, then

$$
H_{k}\left(\bar{Y}_{\mathcal{G}}\right)[A] \cong H_{k}\left(\bar{Y}_{\mathcal{G}^{\prime}}\right)[A]
$$

and otherwise we have the long exact sequence

$$
\cdots \longrightarrow H_{*}\left(\bar{Y}_{\left.\mathcal{G}\right|_{G} \oplus \mathcal{G} / G}\right)[G \oplus A / G] \stackrel{\phi_{G}}{\longrightarrow} H_{*}\left(\bar{Y}_{\mathcal{G}}\right)[A] \longrightarrow H_{*}\left(\bar{Y}_{\mathcal{G}^{\prime}}\right)[A] \longrightarrow \cdots,
$$

where the connecting map is induced by the composition

$$
H_{*}\left(\bar{Y}_{\mathcal{G}^{\prime}}\right) \longrightarrow H_{*}\left(\bar{Y}_{\mathcal{G}^{\prime}} \mid d_{G}^{\prime}\right) \cong H_{*}\left(N_{d_{G}^{\prime}}\left(\mathcal{G}^{\prime}\right) \mid d_{G}^{\prime}\right) \longrightarrow H_{*-1}\left(d_{G}\right),
$$

where $H_{*}(X \mid Y):=H_{*}(X, X \backslash Y)$ and the last map is induced from the morphism $H_{*}\left(\mathbb{R}^{k} \mid\{0\}\right) \cong H_{*-1}\left(S^{k-1}\right) \rightarrow H_{*-1}\left(\mathbb{P}^{k-1}\right)$.

Proof. The key observation is that by Corollary 2.8, the map

$$
d_{G}=\bar{Y}_{\left.\mathcal{G}\right|_{G} \oplus \mathcal{G} / G} \longrightarrow d_{G}^{\prime}=\bar{Y}_{\left.\mathcal{G}^{\prime}\right|_{G} \oplus \mathcal{G} / G}
$$

has a section, and thus the mapping cone long exact sequence breaks up into split short exact sequences

$$
0 \longrightarrow H_{*+1}\left(d_{G} \longrightarrow d_{G}^{\prime}\right) \longrightarrow H_{*}\left(d_{G}\right) \longrightarrow H_{*}\left(d_{G}^{\prime}\right) \longrightarrow 0
$$


We may thus identify the mapping cone homology with its image in $H_{*}\left(d_{G}\right)$ :

$$
H_{k+1}\left(d_{G} \longrightarrow d_{G}^{\prime}\right) \cong \sum_{A \in \mathcal{G} / G} H_{k}\left(\bar{Y}_{\left.\left.\mathcal{G}\right|_{G} \oplus \mathcal{G} / G\right)}\right)[G \oplus A] .
$$

The identification of the map

$$
H_{*+1}\left(d_{G} \longrightarrow d_{G}^{\prime}\right) \longrightarrow H_{*}\left(\bar{Y}_{\mathcal{G}}\right)
$$

follows from functoriality and the commutative diagram

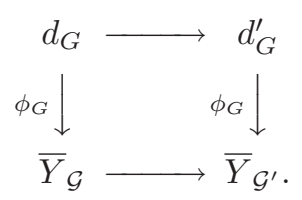

The remainder of the proof is straightforward.

If $G$ is minimal in $\mathcal{G}$ (which is the only case that need be considered when constructing De Concini-Procesi models via repeated blow-ups), we find that the projective bundle is trivial, and thus we can (noncanonically) compute the cohomology of the exceptional divisor via the Künneth formula. We find

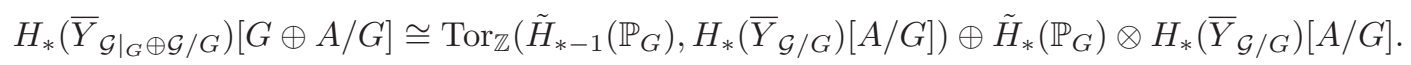

Since the torsion subgroup of $\tilde{H}_{*-1}\left(\mathbb{P}_{G}\right)$ has exponent 2 , the Tor $_{\mathbb{Z}}$ component consists entirely of 2-torsion; thus modulo that 2-torsion, we obtain the canonical isomorphism

$$
\begin{aligned}
& 2 H_{*+\operatorname{dim}(G)-1}\left(\bar{Y}_{\left.\left.\mathcal{G}\right|_{G} \oplus \mathcal{G} / G\right)}\right)[G \oplus A / G] \\
& \quad \cong \begin{cases}H_{\operatorname{dim}(G)-1}\left(\mathbb{P}_{G}\right) \otimes 2 H_{*}\left(\bar{Y}_{\mathcal{G} / G}\right)[A / G], & \operatorname{dim}(G)=0(2), \\
0, & \operatorname{dim}(G)=1(2) .\end{cases}
\end{aligned}
$$

\section{A cell structure for $\bar{Y}_{\mathcal{G}}$}

We will construct the isomorphism of the main theorem via a chain map; this will require a careful choice of complex for the homology of $\bar{Y}_{\mathcal{G}}$.

To each forest $F$ in $\mathcal{G}$ with root $\sum_{G \in \mathcal{G}} G$, we may associate a corresponding composition of operad maps:

$$
\phi_{F}: \prod_{i} \bar{Y}_{\left(\left.\mathcal{G}\right|_{F_{i}}\right) / \operatorname{child}_{F}\left(F_{i}\right)} \longrightarrow \bar{Y}_{\mathcal{G}} .
$$

Indeed, if $C$ is any sum of independent elements of $F$, we can take the composition

$$
\phi_{C} \circ \phi_{\left.F\right|_{C}} \times \phi_{F / C}
$$

where $\left.F\right|_{C}$ and $F / C$ are the induced forests in $\left.\mathcal{G}\right|_{C}$ and $\mathcal{G} / C$. It then follows easily from the composition law that this composition is independent of $C$. Since each $\phi_{C}$ is injective, it follows that $\phi_{F}$ is injective for all $F$, and it is thus reasonable to consider the image $d_{F}$ of $\phi_{F}$. In particular, if $F$ consists only of (the components of) its root, then $d_{F}=\bar{Y}_{\mathcal{G}}$, while if $F$ consists only of the element $G$ in addition to its root, then $d_{F}=d_{G}$.

Now, suppose $\mathcal{C}_{\mathcal{G}}$ has maximal element $\operatorname{root}(\mathcal{G})=G_{1} \oplus G_{2} \oplus \ldots \oplus G_{k}$ of dimension $d$. For each $0 \leqslant i \leqslant d-k$ (the dimension of $\bar{Y}_{\mathcal{G}}$ ), let $X^{i}$ be the union of $d_{F}$ for all forests $F$ with $\operatorname{root}(F)=\operatorname{root}(\mathcal{G})$ and $d-i$ nodes. 
Theorem 5.1. Suppose every chain in $\mathcal{C}_{\mathcal{G}}$ can be extended to a complete flag (that is, with all codimensions 1$)$. Then, for all $n$,

$$
H_{*}\left(X^{n+1}, X^{n}\right)
$$

is free, supported in degree $n+1$. Moreover, the induced map

$$
H_{n+1}\left(X^{n+1}, X^{n}\right) \longrightarrow H_{n}\left(X^{n}\right) \longrightarrow H_{n}\left(X^{n}, X^{n-1}\right)
$$

provides these groups with a chain complex structure, with associated homology groups canonically isomorphic to $H_{*}\left(\bar{Y}_{\mathcal{G}}\right)$.

In other words, the sequence $X^{0} \subset X^{1} \subset \ldots \subset X^{d-k}$ behaves homologically as the sequence of skeletons of a CW complex. (We conjecture that this is in fact the sequence of skeletons of a regular cell complex, but the homological statement will suffice for our purposes.)

Proof of Theorem 5.1. It suffices to prove the claim about $H_{*}\left(X^{i+1}, X^{i}\right)$, since then the derivation of cellular homology for $\mathrm{CW}$ complexes carries over mutatis mutandum. Now, it was shown in [4] that the intersection of $d_{F}$ and $d_{F^{\prime}}$ is the submanifold $d_{F \cup F^{\prime}}$ if $F \cup F^{\prime}$ is a forest, and empty otherwise. We thus have the canonical isomorphism

$$
H_{*}\left(X^{n+1}, X^{n}\right)=\bigoplus_{|F|=d-n-1} H_{*}\left(d_{F}, d_{F} \cap X^{n}\right) .
$$

But then pulling this back through $\phi_{F}$ reduces to the case $|F|=k$; that is, $H_{*}\left(\bar{Y}_{\mathcal{G}}, \bigcup_{G \in \mathcal{G}} d_{G}\right)$.

Now, by the hypotheses, each $G$ contains a 1-dimensional space in the building set, or equivalently $G^{\perp}$ is contained in a hyperplane. In other words, $\bar{Y}_{\mathcal{G}} / \bigcup_{G \in \mathcal{G}} d_{G}$ is homeomorphic to the quotient of $\prod_{i} \mathbb{P}_{G_{i}}$ by a nonempty hyperplane arrangement. But this in turn is homeomorphic to a wedge of spheres (one-point compactifications of intersections of open half-spaces).

Remark. Note that the hypothesis is necessary. Indeed, suppose $F$ were a maximal forest with $d-i$ nodes for some $i>0$ (guaranteed to exist if the hypothesis fails). Then $d_{F} \subset X^{i}$ is disjoint from $X^{i-1}$, and thus its contribution to $H_{*}\left(X^{i}, X^{i-1}\right)$ is simply $H_{*}\left(d_{F}\right)$. But $H_{0}\left(d_{F}\right)=$ $\mathbb{Z} \neq 0$.

With this in mind, we will denote the above chain complex as $C_{*}\left(\bar{Y}_{\mathcal{G}}\right)$, and say that a building set $\mathcal{G}$ satisfying the hypotheses is 'cellular'.

For our purposes, the hypotheses are not particularly onerous; we can simply adjoin generic hyperplanes until they hold. This, of course, leads to the danger that constructions based on the cellular chain complex might depend on the choice of hyperplanes. To control this, we may use the chain map from the following trivial lemma. Note that the map goes in the reverse direction to the usual case of a morphism adjoining a subspace to a building set.

Lemma 5.2. Suppose $\mathcal{G}^{\prime}=\mathcal{G} \cup\{H\}$ with $\operatorname{dim}(H)=1$ and $\mathcal{G}$ cellular. Then the identity $\operatorname{map} \bar{Y}_{\mathcal{G}} \rightarrow \bar{Y}_{\mathcal{G}^{\prime}}$ is cellular.

It will also be helpful to have at our disposal various special cases of operad maps that respect the cell structure. The easiest is the case of a purely operadic weak morphism.

Lemma 5.3. Let $\mathcal{G}$ be a cellular building set, and $C \in \mathcal{C}_{\mathcal{G}}$. Then $\left.\mathcal{G}\right|_{C}$ and $\mathcal{G} / C$ are cellular, as is the operad map $\phi_{C}$. The associated map on the cellular chain complex is injective. 
Proof. Let $F$ be a $\left.\mathcal{G}\right|_{C} \oplus \mathcal{G} / C$-forest with root $C \oplus \operatorname{root}(\mathcal{G} / C)$. Then (essentially by definition) $\phi_{C} \circ \phi_{F}=\phi_{F^{\prime}}$ for some $\mathcal{G}$-forest containing $C$, and this correspondence is bijective. In particular, $d_{F}$ maps isomorphically to $d_{F^{\prime}}$, and any extension of $F^{\prime}$ to a $d$-node forest induces such an extension of $F$. The claim follows.

REMARK. Thus, more generally, the maps $\phi_{F}$ are all cellular (hardly surprising) and act injectively on the chain complex.

Lemma 5.4. Let $\mathcal{G}^{\prime} \subset \mathcal{G}$ be cellular building sets in $V$. If, for all $G \in \mathcal{G}, G \cap \operatorname{root}\left(\mathcal{G}^{\prime}\right) \in \mathcal{C}_{\mathcal{G}^{\prime}}$, then the induced (surjective) morphism $\phi_{\iota}: \bar{Y}_{\mathcal{G}} \rightarrow \bar{Y}_{\mathcal{G}^{\prime}}$ is cellular. Moreover, the induced map on the cellular chain complex is surjective.

Proof. We find by a straightforward induction that, for any $\mathcal{G}$-forest $F$ with $\operatorname{root} \operatorname{root}(\mathcal{G})$, the image of $d_{F}$ under $\phi_{\iota}$ is of the form $d_{F^{\prime}}$, where $F^{\prime}$ is the $\mathcal{G}^{\prime}$-forest consisting of all components of all spaces $G \cap \operatorname{root}\left(\mathcal{G}^{\prime}\right)$ for $G \in F$. The induced morphism

$$
H_{\operatorname{dim}\left(d_{F}\right)}\left(d_{F}, d_{F} \cap X^{\operatorname{dim}\left(d_{F}\right)-1}\right) \longrightarrow C_{\operatorname{dim}\left(d_{F}\right)}\left(\bar{Y}_{\mathcal{G}^{\prime}}\right)
$$

is thus 0 unless $\operatorname{dim}\left(d_{F}\right)=\operatorname{dim}\left(d_{F^{\prime}}\right)$, in which case it is given by the isomorphism

$$
H_{\operatorname{dim}\left(d_{F}\right)}\left(d_{F}, d_{F} \cap X^{\operatorname{dim}\left(d_{F}\right)-1}\right) \cong H_{\operatorname{dim}\left(d_{F}^{\prime}\right)}\left(d_{F}^{\prime}, d_{F}^{\prime} \cap X^{\operatorname{dim}\left(d_{F}^{\prime}\right)-1}\right) .
$$

It therefore only remains to show that any $\mathcal{G}^{\prime}$-forest $F^{\prime}$ with $\operatorname{root} \operatorname{root}\left(\mathcal{G}^{\prime}\right)$ can be obtained as the image of some $\phi_{F}$ of the same dimension. But this is straightforward: we may extend any forest chain associated to $F^{\prime}$ by including a complete chain from $\operatorname{root}\left(\mathcal{G}^{\prime}\right)$ to $\operatorname{root}(\mathcal{G})$, and thus obtain a forest chain with associated forest $F$.

Remark. Suppose $\mathcal{G}^{\prime}$ is a cellular building set, and let $G \in V$ be such that $\mathcal{G}=\mathcal{G}^{\prime} \cup\{G\}$ is a building set. If $G \in \mathcal{C}_{\mathcal{G}^{\prime}}$, then $\mathcal{G}$ is cellular, and the lemma applies. Otherwise, since $\mathcal{G}^{\prime} \cup\{G\}$ is a building set, any minimal element of $\mathcal{C}_{\mathcal{G}^{\prime}}$ containing $G$ is indecomposable, and thus, for a generic $v \in G, \mathcal{G}^{\prime} \cup\{\langle v\rangle\}$ and $\mathcal{G} \cup\{\langle v\rangle\}$ are building sets. By induction on $\operatorname{dim} \operatorname{root}\left(\mathcal{C}_{\left.\mathcal{G}^{\prime}\right|_{G}}\right)$, we eventually obtain a pair of building sets satisfying the hypotheses of the lemma. It follows that any map $\phi_{\iota}$ can be written as a product of morphisms each of which is cellular for some cell structure of the kind we are considering.

Although $\bar{Y}_{\mathcal{G}}$ is not in general orientable, we can still define a fundamental class in

$$
H_{d-k}\left(\bar{Y}_{\mathcal{G}}, X^{d-k-1}\right)
$$

as long as the components of the maximal element of $\mathcal{C}_{\mathcal{G}}$ are even-dimensional. Indeed, we then have a natural map

$$
\begin{aligned}
\bigotimes_{i} \operatorname{or}\left(G_{i}\right) & \cong H_{d-k}\left(\prod_{i} \mathbb{P}_{G_{i}}\right) \longrightarrow H_{d-k}\left(\prod_{i} \mathbb{P}_{G_{i}},\left(\prod_{i} \rho_{G_{i}}\right)\left(X^{d-k-1}\right)\right) \\
& \cong H_{d-k}\left(\bar{Y}_{\mathcal{G}}, X^{d-k-1}\right) .
\end{aligned}
$$

More generally, given an ordered 2-divisible forest $F$ with $\operatorname{root}(F)=\operatorname{root}(\mathcal{G})$, we obtain a map

$$
\mu_{F}: \operatorname{or}(\operatorname{root}(\mathcal{G})) \longrightarrow H_{*}\left(d_{F}, d_{F} \cap X^{d-|F|-1}\right)
$$

by composing a map of the above form with $\phi_{F}$. 
Theorem 5.5. Let $\mathcal{G}$ have root $A$, and fix a class $\omega \in \operatorname{or}(A)$. Then the map $\mu: F \mapsto \mu_{F}(\omega)$ gives a well-defined map

$$
\mu: C_{f}^{\operatorname{dim}(A)-*}(A) \longrightarrow C_{*}\left(\bar{Y}_{\mathcal{G}}\right)
$$

which is operadic with respect to purely operadic weak morphisms $\phi_{C}$ with $C$ 2-divisible, and satisfies

$$
\partial \mu(F)=2 \mu(d F)
$$

The corresponding map on homology is independent of the choice of hyperplanes making $\mathcal{G}$ cellular.

Proof. The fact that $\mu$ is operadic with respect to purely operadic weak morphisms follows essentially immediately from its definition. In particular, we may pull the boundary computation back through $\phi_{F}$, and thus reduce to the case that $F$ consists only of root nodes. But then the product structure of $\bar{Y}_{\mathcal{G}}$ allows us to reduce to the case that $F$ consists of the single node $V^{*}$.

Since the cellular chain complex splits as a direct sum over forests, we need only determine the induced map

$$
H_{n}(\mathbb{P}(V)) \longrightarrow H_{n}\left(\bar{Y}_{\mathcal{G}}, X^{n-1}\right) \stackrel{\partial}{\longrightarrow} H_{n-1}\left(d_{G}, d_{G} \cap X^{n-2}\right)
$$

for $G \in \mathcal{G} \backslash\left\{V^{*}\right\}$, where $n=\operatorname{dim}(V)-1$. Let $\pi$ be the natural map $\bar{Y}_{\mathcal{G}} \rightarrow \bar{Y}_{\left\{G, V^{*}\right\}}$. Then we have a commutative diagram

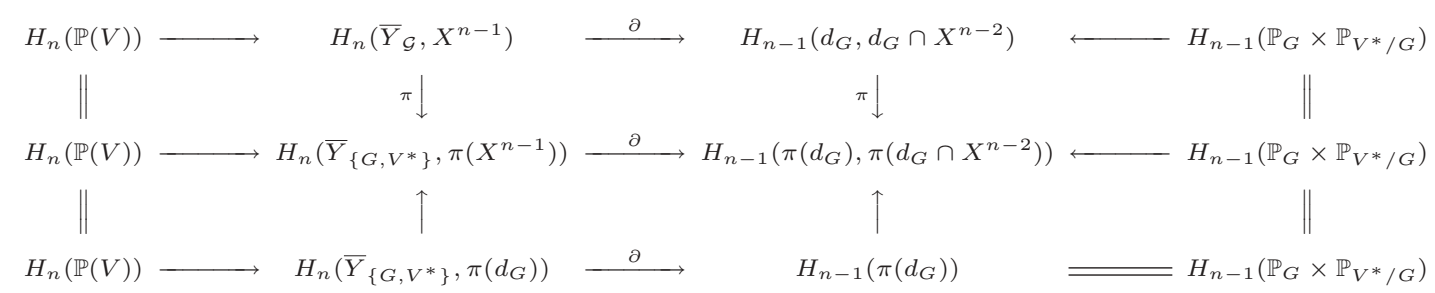

in which the first row of vertical arrows consists of isomorphisms. In particular, it suffices to determine the action of $\partial$ in the bottom row, which is straightforward.

That the action on homology is independent of the choice of hyperplanes follows immediately from the fact that the maps $\mu_{F}$ commute with the adjunction-of-hyperplanes maps.

With this in mind, let $H_{f}^{*}(A ; 2 d)$ denote the cohomology of the forest complex with differential multiplied by 2 . Note that the only effect of this is to extend the cohomology by some 2 -torsion:

$$
2 H_{f}^{*}(A ; 2 d) \cong H_{f}^{*}(A) .
$$

Since we will be focusing on the root, it will be notationally convenient to define $H_{f}^{*}(\mathcal{G} ; 2 d):=$ $H_{f}^{*}(\operatorname{root}(\mathcal{G}) ; 2 d)$, and similarly for chain complexes.

Lemma 5.6. Let $G \in \mathcal{G}$ be such that $\mathcal{G}^{\prime}=\mathcal{G} \backslash\{G\}$ is a cellular building set with $G \in \mathcal{C}_{\mathcal{G}^{\prime}}$. Then, for any 2-divisible $\mathcal{G}$-forest $F,\left(\phi_{\iota}\right)_{*}(\mu(F))$ can be computed as follows. If $G \notin F$, then $\left(\phi_{\iota}\right)_{*}(\mu(F))=\mu(F)$. If $G \in F$ and there is a unique $\mathcal{G}^{\prime}$-component $G^{\prime}$ of $G$ which is not a component of child $F(G)$, then $\left(\phi_{\iota}\right)_{*}(\mu(F))=\mu\left(F^{\prime}\right)$ where $F^{\prime}$ is the (2-divisible) forest obtained from $F$ by replacing $G$ by $G^{\prime}$. In all remaining cases, $\left(\phi_{\iota}\right)_{*}(\mu(F))=0$. 
Proof. This follows easily by a consideration of the composition $\phi_{\iota} \circ \phi_{F}$, using the fact that

$$
\phi_{\iota} \circ \phi_{C}= \begin{cases}\phi_{C} \circ\left(\phi_{\iota} \times 1\right), & G \subset C, \\ \phi_{C} \circ\left(1 \times \phi_{\iota}\right), & G \not \subset C ;\end{cases}
$$

moreover in the second case, the morphism $\iota$ is the identity whenever $G+C \in \mathcal{G}^{\prime} / C$. The claim when $G \notin F$ is thus immediate.

For $G \in F$, we may take $C=G$ and thus reduce to considering the case $G=\operatorname{root}(F)$. In that case, taking $C=\operatorname{child}_{F}(G)$ above, if $G=\operatorname{child}_{F}(G)+G^{\prime}$ for some $G^{\prime} \in \mathcal{G}^{\prime}$, then we may take $G^{\prime}$ to be a $\mathcal{G}^{\prime}$-component of $G$ (the unique such component not a component of $\operatorname{child}_{F}(G)$ ), and since $G^{\prime} / C=G / C$, we obtain $\phi_{F^{\prime}}$ as desired. Note that since $G^{\prime}$ is a component of $G$ and all other components of $G$ are even-dimensional, the same applies to $G^{\prime}$; in addition, since $G$ is a sum of subspaces in $F^{\prime}$, the root of $F^{\prime}$ is unchanged.

Otherwise, if $G \in F$ but $G$ has multiple components not contained in $\operatorname{child}_{F}(G)$, then we reduce to the case $F=\{G\}$, for which triviality of the map follows by dimension considerations.

Lemma 5.7. Let $\mathcal{G}$ be a building set with root $A$. The image in homology of the map $\mu$ is contained in $H_{*}\left(\bar{Y}_{\mathcal{G}}\right)[A]$.

Proof. We need to show that, for any $C \in \mathcal{C}_{\mathcal{G}}$ not equal to $A, \pi_{C} \circ \mu=0$. This is a slight difficulty, given that we may not be able to arrange for $\pi_{C}$ itself to be cellular. However, we can always write $\pi_{C}$ as a composition of maps $\phi_{\iota}$, each of which removes a single element of $\mathcal{G}$. Each of those maps can be made cellular (and made to satisfy the hypotheses of the lemma) by adjoining suitably generic hyperplanes, and the action thus commutes with $\mu$. At some point in the chain of maps, the root of the building set necessarily decreases; we claim that at that point, the image of $\mu$ is annihilated. But this follows from the fact that for such a map, $\left(\phi_{\iota}\right)_{*}(\mu(F))=0$ for all 2-divisible $\mathcal{G}$-forests $F$ with $\operatorname{root} A$.

THEOREM 5.8. The map $\mu$ is compatible with the blow-up long exact sequence as follows. Suppose $G \in \mathcal{G}$, that $\mathcal{G}^{\prime}:=\mathcal{G} \backslash\{G\}$ is a building set, and that $G \notin \mathcal{C}_{\mathcal{G}^{\prime}}$, but $G \subset \operatorname{root}\left(\mathcal{G}^{\prime}\right)$. Then we have the following commutative diagram of long exact sequences:

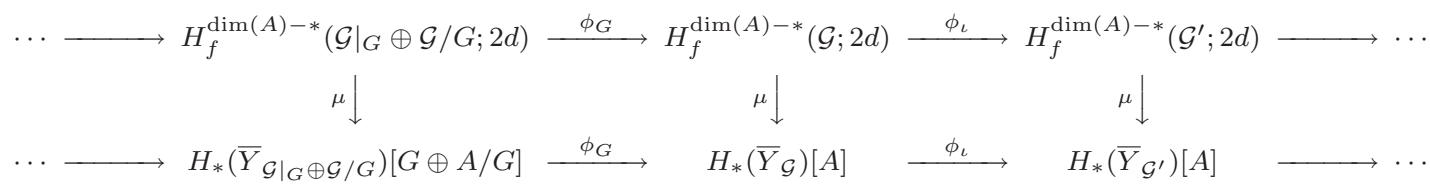

Proof. First, by adjoining sufficient hyperplanes to $\mathcal{G}$, we may arrange for the maps to be cellular; in particular, this means that now $G \in \mathcal{C}_{\mathcal{G}^{\prime}}$, but with at least one hyperplane component.

Now, the top row is the long exact sequence associated to the short exact sequence

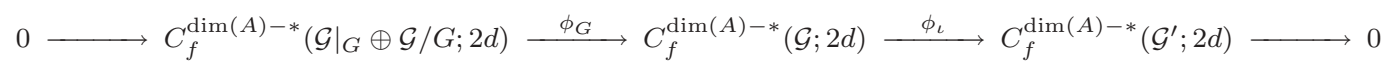

of cochain complexes; we will thus be able to obtain the desired diagram if we can exhibit a corresponding short exact sequence for the bottom row making everything commute.

On the bottom row, it is tempting to consider the sequence

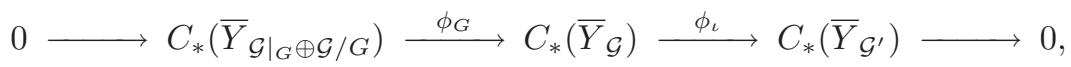


which indeed forms a commutative diagram with the sequence on forest cochains. Unfortunately, this sequence is not exact in the middle; in fact, $\phi_{\iota} \circ \phi_{G} \neq 0$. However, we do have the inclusion $\operatorname{ker} \phi_{\iota} \subset \operatorname{im} \phi_{G}$, and can verify that $\phi_{\iota} \circ \phi_{G} \circ \mu=0$. We thus obtain the commutative diagram

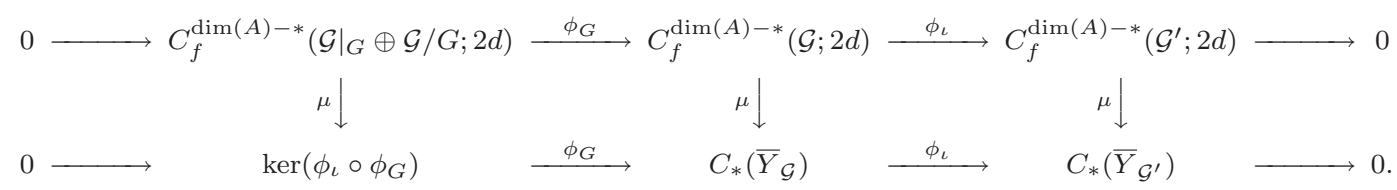

Now, we have the composition $\phi_{\iota} \circ \phi_{G}=\phi_{G} \circ \pi_{A / G}$ (note that $\pi_{A / G}:\left.\mathcal{G}\right|_{G} \times \mathcal{G} / G \rightarrow \mathcal{G} / G$ is cellular) and thus $\operatorname{ker}\left(\phi_{\iota} \circ \phi_{G}\right)=\operatorname{ker}\left(\pi_{A / G}\right)$. The claim follows.

We can now prove a significant portion of the main theorem.

Theorem 5.9. Let $\mathcal{G}$ be a building set. Then the maps

$$
\mu: H_{f}^{\operatorname{dim}(C)-*}\left(\left.\mathcal{G}\right|_{C} ; 2 d ; \mathbb{Z}[1 / 2]\right) \otimes \operatorname{or}(C) \longrightarrow H_{*}\left(\bar{Y}_{\left.\mathcal{G}\right|_{C}} ; \mathbb{Z}[1 / 2]\right)[C] \cong H_{*}\left(\bar{Y}_{\mathcal{G}} ; \mathbb{Z}[1 / 2]\right)[C]
$$

induce an isomorphism

$$
\bigoplus_{C \in \mathcal{C}_{\mathcal{G}}} H_{f}^{\operatorname{dim}(C)-*}\left(\left.\mathcal{G}\right|_{C} ; 2 d ; \mathbb{Z}[1 / 2]\right) \otimes \operatorname{or}(C) \cong H_{*}\left(\bar{Y}_{\mathcal{G}} ; \mathbb{Z}[1 / 2]\right) .
$$

Proof. It suffices to consider the case $C=\operatorname{root}(\mathcal{G})$. If $\mathcal{G}$ consists precisely of the components of $C$ (so $\bar{Y}_{\mathcal{G}}$ is a product of projective spaces), then the isomorphism is immediate; the claim in general follows by induction from Theorem 5.8 and the five-lemma.

\section{2-adic homology}

Of course, we expect (according to the main theorem) that $\mu$ should induce an isomorphism

$$
\bigoplus_{C \in \mathcal{C}_{\mathcal{G}}} 2 H_{f}^{\operatorname{dim}(C)-*}\left(\left.\mathcal{G}\right|_{C} ; 2 d ; \mathbb{Z}\right) \otimes \operatorname{or}(C) \cong 2 H_{*}\left(\bar{Y}_{\mathcal{G}} ; \mathbb{Z}\right)
$$

Since we have shown this over $\mathbb{Z}[1 / 2]$, it only remains to consider the case of 2-adic coefficients. The difficulty here is that the five-lemma does not apply if one weakens 'isomorphism' to 'isomorphism modulo 2-torsion'. Notably, the 2-torsion being ignored by the known maps could easily combine to form 4 -torsion in the unknown (middle) map. If the middle map induces an isomorphism modulo 2-torsion on mod 4 homology, this cannot happen; this suggests that it should suffice for us to consider the action of $\mu$ on mod 4 homology.

In fact, it is not necessary to use the blow-up long exact sequence to reduce to mod 4 homology; one can simply use the following completely general lemma.

Lemma 6.1. Let $\rho: C_{*} \rightarrow D_{*}$ be a chain map of 2-adic chain complexes. If $\rho$ induces an isomorphism

$$
2 H_{*}(C \otimes \mathbb{Z} / 4 \mathbb{Z}) \longrightarrow 2 H_{*}(D \otimes \mathbb{Z} / 4 \mathbb{Z}),
$$

then it induces an isomorphism

$$
2 H_{*}(C) \longrightarrow 2 H_{*}(D)
$$


Proof. Recall that there is a singly graded spectral sequence (the Bockstein spectral sequence) with $r$ th page

$$
B_{n}^{r}(C)=2^{r-1} H_{n}\left(C \otimes \mathbb{Z} / 2^{r} \mathbb{Z}\right)
$$

for $r \geqslant 1$. The hypothesis states that $\rho$ induces an isomorphism $B_{*}^{2}(C) \cong B_{*}^{2}(D)$; since $\rho$ is a chain map, this is an isomorphism of spectral sequences, and thus $\rho$ induces an isomorphism $B_{*}^{r}(C) \cong B_{*}^{r}(D)$ for all $r \geqslant 2$.

Now, since the short exact sequence from the universal coefficient theorem is split, it follows that it remains exact when multiplied by a power of 2 . In particular, we have a (split) short exact sequence

$$
0 \longrightarrow 2^{r-1}\left(H_{n}(C) \otimes \mathbb{Z} / 2^{r} \mathbb{Z}\right) \longrightarrow 2^{r-1} H_{n}\left(C \otimes \mathbb{Z} / 2^{r} \mathbb{Z}\right) \longrightarrow 2^{r-1} \operatorname{Tor}\left(H_{n-1}(C), \mathbb{Z} / 2^{r} \mathbb{Z}\right) \longrightarrow 0 .
$$

Now, suppose that we know that $\rho$ induces an isomorphism $2 H_{n-1}(C) \cong 2 H_{n-1}(D)$ (certainly the case for $n \leqslant 0$ ). Then it follows from the universal coefficient exact sequence that it induces isomorphisms

$$
\begin{aligned}
2^{r-2}\left(\left(2 H_{n}(C)\right) \otimes \mathbb{Z} / 2^{r-1} \mathbb{Z}\right) & =2^{r-1}\left(H_{n}(C) \otimes \mathbb{Z} / 2^{r} \mathbb{Z}\right) \cong 2^{r-1}\left(H_{n}(D) \otimes \mathbb{Z} / 2^{r} \mathbb{Z}\right) \\
& =2^{r-2}\left(\left(2 H_{n}(D)\right) \otimes \mathbb{Z} / 2^{r-1} \mathbb{Z}\right)
\end{aligned}
$$

for all $r \geqslant 2$. But then, by the five-lemma, $\rho$ induces an isomorphism $2 H_{n}(C) \cong 2 H_{n}(D)$ as required, and the result follows by induction.

Now, $2\left(H_{*}(C \otimes \mathbb{Z} / 4 \mathbb{Z})\right)$ can be computed as follows: let

$$
\beta: H_{*}\left(C \otimes \mathbb{F}_{2}\right) \longrightarrow H_{*-1}\left(C \otimes \mathbb{F}_{2}\right)
$$

be the Bockstein morphism, that is, the connecting map in the long exact sequence

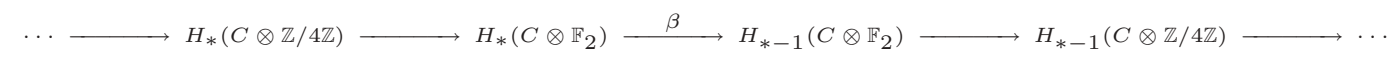

Then $\beta$ makes $H_{*}\left(C \otimes \mathbb{F}_{2}\right)$ a chain complex, and

$$
2 H_{*}(C \otimes \mathbb{Z} / 4 \mathbb{Z}) \cong H_{*}\left(H_{*}\left(C \otimes \mathbb{F}_{2}\right) ; \beta\right) .
$$

In particular, we may restate the lemma by saying that $\rho$ induces an isomorphism modulo 2 -torsion on homology if and only if it induces an isomorphism on $\beta$-homology.

We thus need to understand the $\bmod 2$ homology of $\bar{Y}_{\mathcal{G}}$, and the associated Bockstein morphism. Now, since $\bar{Y}_{\mathcal{G}}$ is a smooth real algebraic variety, every smooth subvariety induces a class in mod 2 homology (since issues of orientability can be ignored). In general, such classes do not span homology, but in our case, they do suffice; that is, $\bar{Y}_{\mathcal{G}}$ is 'algebraically maximal' in the sense of [14]. This follows, for instance, from the fact $[4]$ that the cohomology of $\bar{Y}_{\mathcal{G}}(\mathbb{C})$ is generated by $\mathbb{R}$-rational cycles. (This can also be seen directly from Proposition 6.2 below, which gives an explicit basis of classes associated to subvarieties.)

This allows us to extend the construction of the fundamental class to classes of other degrees. If $V^{*} \in \mathcal{G}$ and $\operatorname{dim}(V)=d$, we construct a canonical class in $H_{i}\left(\bar{Y}_{\mathcal{G}} ; \mathbb{F}_{2}\right)$ as follows. Choose a generic $(i+1)$-dimensional subspace of $V^{*}$, and consider the closure of its image in $\bar{Y}_{\mathcal{G}}$. The result is an $i$-dimensional subvariety, and any two such subvarieties are clearly homotopic; we thus obtain the desired canonical homology class. Moreover, we readily verify that if $i>0$, this class is actually in $H_{i}\left(\bar{Y}_{\mathcal{G}} ; \mathbb{F}_{2}\right)\left[V^{*}\right]$.

More generally, let $F$ be a forest (not necessarily 2-divisible) with $\operatorname{root} \operatorname{root}(\mathcal{G})$, and suppose we are also given a map $d: F \rightarrow \mathbb{Z}^{+}$such that $d(G)<\operatorname{dim}(G)-\operatorname{dim}\left(\operatorname{child}_{F}(G)\right)$ for all $G \in F$. Then we have a canonical class in

$$
H_{d(G)}\left(\left(\left.\mathcal{G}\right|_{G}\right) / \operatorname{child}_{F}(G) ; \mathbb{F}_{2}\right)
$$


for all $G \in F$, and may define $\mu(F, d)$ to be the image under $\phi_{F}$ of the product of these classes. Note that this is compatible with our previous notation, in the sense that if $F$ is 2-divisible and $d_{F}(G)=\operatorname{dim}(G)-\operatorname{dim}\left(\operatorname{child}_{F}(G)\right)-1$, then $\mu\left(F, d_{F}\right)=\mu(F) \otimes \mathbb{F}_{2}$.

Proposition 6.2. The classes $\mu(F, d)$ for $\kappa(F, d):=\sum_{G \in F} d(G)=k$ form a basis of $H_{k}\left(\bar{Y}_{\mathcal{G}} ; \mathbb{F}_{2}\right)[\operatorname{root}(\mathcal{G})]$.

Proof. Let $A:=\operatorname{root}(\mathcal{G})$. Modulo 2, the blow-up exact sequence decomposes into short exact sequences

$$
0 \longrightarrow H_{*}\left(\bar{Y}_{\left.\mathcal{G}\right|_{G} \oplus \mathcal{G} / G} ; \mathbb{F}_{2}\right)[G \oplus A / G] \stackrel{\phi_{G}}{\longrightarrow} H_{*}\left(\bar{Y}_{\mathcal{G}} ; \mathbb{F}_{2}\right)[A] \longrightarrow H_{*}\left(\bar{Y}_{\mathcal{G}^{\prime}} ; \mathbb{F}_{2}\right)[A] \longrightarrow 0
$$

where we recall that $G \in \mathcal{G}$ such that $\mathcal{G}^{\prime}:=\mathcal{G} \backslash\{G\}$ is a building set and $G \notin \mathcal{C}_{\mathcal{G}^{\prime}}$; the connecting map is trivial by Corollary 4.4. We moreover readily verify that if $G \in F$, so there is a class

$$
\mu(F, d) \in H_{*}\left(\bar{Y}_{\left.\left.\mathcal{G}\right|_{G} \oplus \mathcal{G} / G\right)}[G \oplus A / G],\right.
$$

then $\phi_{G}(\mu(F, d))=\mu(F, d)$. Similarly, if $G \notin F$, then $\mu(F, d)$ maps to its counterpart in $H_{*}\left(\bar{Y}_{\mathcal{G}^{\prime}}\right)[A]$. But then the claim follows by induction.

REMARK. The above basis (summed over the graded pieces) is trivially bijective with the basis of $H^{*}\left(\bar{Y}_{\mathcal{G}}(\mathbb{C})\right)$ given in $[\mathbf{1 8}]$, which by the results of $[\mathbf{1 4}]$ alluded to above induces a basis of $H_{*}\left(\bar{Y}_{\mathcal{G}} ; \mathbb{F}_{2}\right)$. However, the precise relation between these bases is somewhat unclear; for instance, Yuzvinsky's basis is not compatible with the natural grading by $\mathcal{C}_{\mathcal{G}}$. Note also that precisely the same argument (the long exact sequence for complex blow-ups also splits into short exact sequences) shows that the above algebraic cycles form a basis of the homology of the complex locus.

Next, we need to determine the action of the Bockstein morphism. For $G \in \mathcal{G}$, we define a $\operatorname{map} \beta_{G}$ by

$$
\beta_{G}(\mu(F, d))=0
$$

if $G \in F, F \cup\{G\}$ is not a forest, or

$$
\operatorname{dim}\left(G / \operatorname{child}_{F \cup\{G\}}(G)\right) \bmod 2=1 .
$$

Otherwise,

$$
\beta_{G}(\mu(F, d))=\mu\left(F \cup\{G\}, d^{\prime}\right)
$$

where

$$
d^{\prime}(H)= \begin{cases}\operatorname{dim}\left(G / \operatorname{child}_{F \cup\{G\}}(G)\right)-1, & H=G, \\ d(H)-\operatorname{dim}\left(G / \operatorname{child}_{F \cup\{G\}}(G)\right), & G \in \operatorname{child} H, \\ d(H) & \text { otherwise }\end{cases}
$$

Lemma 6.3. The Bockstein morphism acts on forest classes $\mu(F, d)$ as follows:

$$
\beta(\mu(F, d))=\sum_{G \in \mathcal{G}} \beta_{G}(\mu(F, d))+\sum_{\substack{G \in F \\ d(G) \bmod 2=0}} \mu\left(F, d-\delta_{G}\right) .
$$

Proof. First suppose that $F=\left\{V^{*}\right\}$ and $d\left(V^{*}\right)=\operatorname{dim}(V)-1$. If $\operatorname{dim}(V)$ is even, then this lifts to the integral chain $\mu\left(\left\{V^{*}\right\}\right)$, and the claim follows from Theorem 5.5; a similar calculation establishes the claim when $\operatorname{dim}(V)$ is odd. 
Now, if $F=\left\{V^{*}\right\}, 0<d\left(V^{*}\right)<\operatorname{dim}(V)-1$, then $\mu(F, d)$ can be calculated using the fact that $\beta$ is functorial, so can be transported through operad maps; in particular, the operad map associated to inclusion of a generic $d\left(V^{*}\right)$-plane. The claim for general $F$ then follows using the map $\phi_{F}$.

Given a pair $(F, d)$, define the defect

$$
\delta(F, d)=|\{G: G \in F \mid d(G) \bmod 2=0\}| .
$$

Of the two components of the above expression for $\beta$, only the second component changes the defect, decreasing it by 1 . We may thus interpret $\beta$ as the differential of the total complex of a suitable double complex. To be precise, for $0 \leqslant p \leqslant q$, define

$$
\begin{aligned}
E_{p, q}^{0} & =\bigoplus_{\substack{\delta(F, d)=p \\
\kappa(F, d)=p+q}} \mathbb{F}_{2} \mu(F, d), \\
\partial_{1}(\mu(F, d)) & =\sum_{\substack{G \in F \\
d(G) \bmod 2=0}} \mu\left(F, d-\delta_{G}\right), \\
\partial_{2}(\mu(F, d)) & =\sum_{G \in \mathcal{G}} \beta_{G}(\mu(F, d)) .
\end{aligned}
$$

Then $\partial_{1}\left(E_{p, q}^{0}\right) \subset E_{p-1, q}^{0}, \partial_{2}\left(E_{p, q}^{0}\right) \subset E_{p, q-1}^{0}$, and we readily verify that this defines a double complex with total complex $\left(H_{k}, \beta\right)$.

Lemma 6.4. The map

$$
\mu: H_{f}^{\operatorname{dim}(C)-*}\left(\mathcal{G} ; 2 d ; \mathbb{F}_{2}\right) \longrightarrow H_{*}\left(\bar{Y}_{\mathcal{G}} ; \mathbb{F}_{2}\right)[\operatorname{root}(\mathcal{G})]
$$

induces an isomorphism on Bockstein homology.

Proof. Since $\mu$ is injective and the image of the chain map $\mu$ is annihilated by $\partial_{1}$ and closed under $\partial_{2}$, it suffices to show that the classes $\mu(F) \in E_{0, q}^{0}$ for 2-divisible forests $F$ are representatives for the $\partial_{1}$-homology on $E_{p, q}^{0}$.

Now, the action of $\partial_{1}$ leaves the forest unchanged, so we may restrict our attention to a single forest $F$; in other words, it suffices to compute the homology of $\partial_{1}$ on the space $\bigoplus_{d} \mu(F, d)$. But this new complex is a product complex, with one factor for each element of $G$. For each $G$, if $\operatorname{dim}(G)-\operatorname{dim}(\operatorname{child}(G))$ is odd, then the homology of the corresponding factor is trivial, and thus $F$ must be 2-divisible. Similarly, if $\operatorname{dim}(G)-\operatorname{dim}(\operatorname{child}(G))$ is even, then the homology is supported in the top degree; the lemma follows.

By Lemma 6.1, this implies that $\mu$ induces an isomorphism modulo 2-torsion on 2-adic homology, which together with Theorem 5.9 implies that $\mu$ induces an isomorphism modulo 2 -torsion on integral homology.

TheOREm 6.5. Let $\mathcal{G}$ be a building set. Then the maps

$$
\mu: H_{f}^{\operatorname{dim}(C)-*}\left(\left.\mathcal{G}\right|_{C} ; 2 d ; \mathbb{Z}\right) \otimes \operatorname{or}(C) \longrightarrow H_{*}\left(\bar{Y}_{\left.\mathcal{G}\right|_{C}} ; \mathbb{Z}\right)[C] \cong H_{*}\left(\bar{Y}_{\mathcal{G}} ; \mathbb{Z}\right)[C]
$$

induce an isomorphism

$$
\bigoplus_{C \in \mathcal{C}_{\mathcal{G}}} 2 H_{f}^{\operatorname{dim}(C)-*}\left(\left.\mathcal{G}\right|_{C} ; 2 d ; \mathbb{Z}\right) \otimes \operatorname{or}(C) \cong 2 H_{*}\left(\bar{Y}_{\mathcal{G}} ; \mathbb{Z}\right)
$$




\section{Operadicity}

To finish proving the main theorem, it only remains to prove that the isomorphism given by $\mu$ is operadic. There is a slight subtlety here, in that $\mu$ is only operadic once we have quotiented out the 2-torsion.

There are, however, three important special cases of operadicity that hold directly on integral homology. First, if $f: V \rightarrow W$ is a morphism such that $f^{*}$ induces a bijection between $\mathcal{G}$ and $\mathcal{G}^{\prime}$, then $\phi_{f}$ is an isomorphism, and commutativity is trivial. Next, if $\mathcal{G}=\mathcal{G}^{\prime} \cup\{G\}$, then we have a commutative diagram

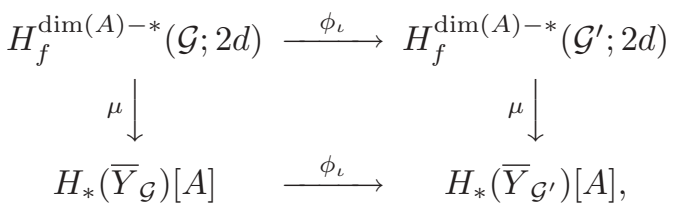

when $A=\operatorname{root}(\mathcal{G})=\operatorname{root}\left(\mathcal{G}^{\prime}\right)$, which immediately implies commutativity of the diagram

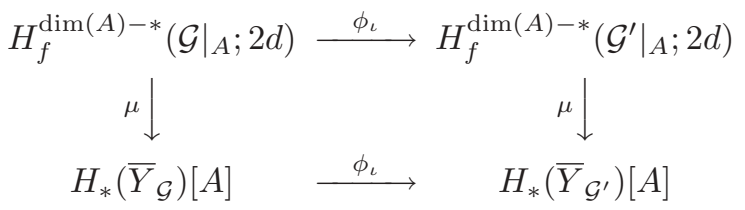

for general $A \in \mathcal{C}_{\mathcal{G}^{\prime}}$; together with the previous case (and the fact that the maps are trivial if $\left.A \notin \mathcal{C}_{\mathcal{G}^{\prime}}\right)$, this shows operadicity whenever $f^{*}: \mathcal{G}^{\prime} \rightarrow \mathcal{G}$ is injective. Finally, if $C \in \mathcal{C}_{\mathcal{G}}$, then we have commutativity of

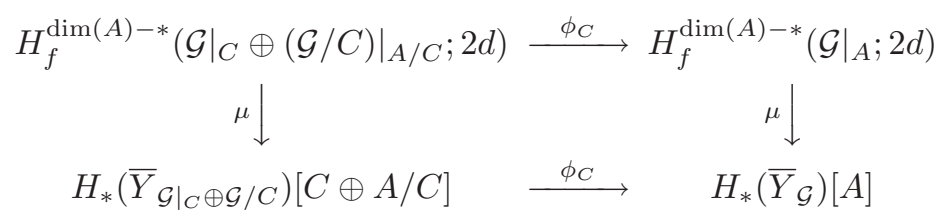

for all $A \supset C$. Composing these three cases, we find that the diagram

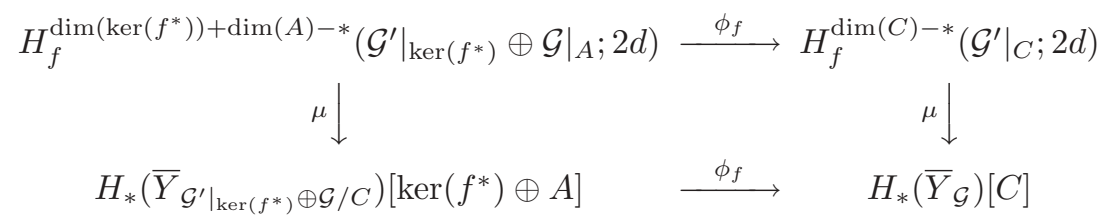

is commutative whenever $\operatorname{ker}\left(f^{*}\right) \in \mathcal{C}_{\left.\mathcal{G}^{\prime}\right|_{C}}$; in particular, if $A \neq f^{*}(C)$, then the horizontal maps are 0 .

Now, in general, given an operad map $f: \mathcal{G} \rightarrow \mathcal{G}^{\prime}$ and spaces $A \in \Pi_{\mathcal{G}}^{(2)}, B, C \in \Pi_{\mathcal{G}^{\prime}}^{(2)}$ with $B \subset \operatorname{ker}\left(f^{*}\right)$, we need to show commutativity of the diagram

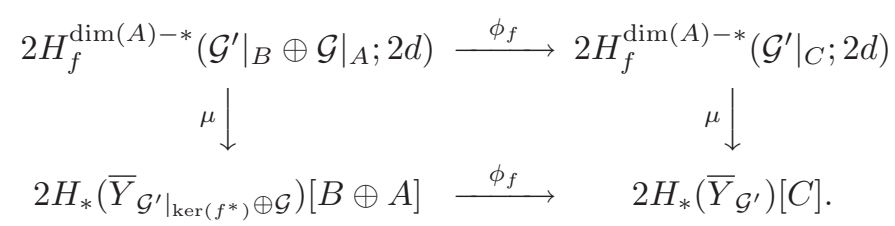

Since $B \subset \operatorname{ker}\left(f^{*}\right)$, we may write $f$ as a composition

$$
f: \mathcal{G} \stackrel{g}{\longrightarrow} \mathcal{G}^{\prime} / B \stackrel{i_{B}}{\longrightarrow} \mathcal{G}^{\prime}
$$


and thus obtain a commutative diagram

$$
\begin{array}{ccc}
2 H_{*}\left(\bar{Y}_{\left.\left.\mathcal{G}^{\prime}\right|_{B} \oplus\left(\mathcal{G}^{\prime} / B\right)\right|_{\operatorname{ker}\left(g^{*}\right)} \oplus \mathcal{G}}\right)[B \oplus 0 \oplus A] & \stackrel{1 \times \phi_{g}}{\longrightarrow} 2 H_{*}\left(\bar{Y}_{\left.\mathcal{G}^{\prime}\right|_{B} \oplus\left(\mathcal{G}^{\prime} / B\right)}\right)[B \oplus C / B] \\
\phi_{B} \times 1 & & \\
2 H_{*}\left(\bar{Y}_{\left.\mathcal{G}^{\prime}\right|_{\text {ker } f^{*}} \oplus \mathcal{G}}\right)[B \oplus A] & \stackrel{\phi_{f}}{\longrightarrow} & 2 H_{*}\left(\bar{Y}_{\mathcal{G}^{\prime}}\right)[C]
\end{array}
$$

of operad maps (the grading on the upper right-hand corner being forced by the requirement that $\phi_{B}$ acts nontrivially). Now, the vertical arrows are respected by $\mu$ (and the left vertical arrow is an isomorphism), so it suffices to show that $\mu$ respects the top row. Since $\mu$ respects products, we find that we may restrict our attention to the case $B=0$. Similarly, we may compose with the map $\phi_{C}$ to restrict to the case $C=\operatorname{root}\left(\mathcal{G}^{\prime}\right)$. If $\operatorname{ker}\left(f^{*}\right)=0$, then $f^{*}: \mathcal{G}^{\prime} \rightarrow \mathcal{G}$ is injective, and we have already verified operadicity. Otherwise, let $H \subset \operatorname{ker}\left(f^{*}\right)$ be a hyperplane, which we may remove from $\mathcal{G}^{\prime}$ if necessary to insure that the corresponding inclusion map is a morphism. Thus if we factor

$$
f: \mathcal{G} \stackrel{g}{\longrightarrow} \mathcal{G}^{\prime} / H \stackrel{i_{H}}{\longrightarrow} \mathcal{G}^{\prime},
$$

then the composition law reads

$$
\phi_{i_{H}} \circ \phi_{g}=\phi_{f} \circ\left(\phi_{\left.i_{H}\right|_{\operatorname{im}(g)}} \times 1\right) .
$$

Since any morphism induces an isomorphism on 0-graded homology, $\phi_{f}$ respects $\mu$ if $\phi_{i_{H}}$ and $\phi_{g}$ both do so.

By induction, it thus only remains to consider operadicity of the morphism $\phi_{i_{H}}$. At the poset level, this map is trivial, so we need simply show that the map

$$
2 H_{*}\left(\bar{Y}_{\mathcal{G}^{\prime} / H}\right)[A] \stackrel{\phi_{i_{H}}}{\longrightarrow} 2 H_{*}\left(\bar{Y}_{\mathcal{G}^{\prime}}\right)[C]
$$

is trivial whenever $H \subset C$. If $A \neq C / H$, this map is trivial even before quotienting by the 2torsion; if $A=C / H$, then one of the two spaces has odd dimension, and thus by Theorem 6.5 that graded piece of the integral homology consists entirely of 2-torsion, so the map is again necessarily trivial.

It follows that $\mu$ is operadic modulo 2-torsion in general, concluding the proof of Theorem 3.7.

\section{Further directions}

In [9], Gaiffi considers the analog of De Concini-Procesi models in which the projective spaces of the definition are replaced by spheres, and shows that the result is a manifold with corners which is homotopy equivalent to the complement of the subspace arrangement. Much of the above structure carries over to this case, with two important exceptions. First, although the resulting structures indeed form a (universal) topological operad, Corollary 2.8 does not hold. To be precise, if $C$ has codimension 1 in $\operatorname{root}(\mathcal{G})$, then the operad map gives rise to two splittings of $\pi_{C}$ which are not homotopic to each other. Thus, in order to obtain a natural grading on homology, it is necessary to assume that this codimension 1 situation never arises. Of course, this condition already appeared in $[\mathbf{5}, \mathbf{1 0}, \mathbf{1 6}, \mathbf{1 9}]$, for similar reasons.

The second, more serious, difficulty is that since we can no longer add hyperplanes without changing the geometry, we do not have a good analog of the cell structure of Section 5 . One way to avoid this would be to generalize the problem by allowing a combination of both projective spaces and spheres in constructing the compactification; at that point, one could again add hyperplanes, so long as they were associated to projective spaces. Presumably, some constraints would be necessary on this association in order to define the operad maps; one likely candidate is that if $\rho_{G}$ maps to a projective space, then so does $\rho_{H}$ for all $H \subset G$. Also, the argument 
of Section 7 would no longer apply, so it might be more difficult to prove that the associated maps were operadic. The relevant poset in this case would presumably be the subposet of $\mathcal{C}_{\mathcal{G}}$ consisting of spaces each component of which is associated to an orientable target $(S(G)$ or $\mathbb{P}(G)$ with $\operatorname{dim}(G)$ even).

One important special case of this would be when $V^{*} \in G$, and only $\rho_{V^{*}}$ maps to a sphere; this is a (nonorientable) double cover of $\bar{Y}_{\mathcal{G}}$ studied for $A_{n-1}$ in [12] and for general Coxeter arrangements in $[\mathbf{1}]$. This is essentially equivalent to studying the homology of the pullback of the orientation sheaf on $\mathbb{P}(V)$, which is straightforward enough using the present methods. We obtain the following.

Theorem 8.1. Let $\mathcal{G}$ be a building set containing $V^{*}$, and let $\omega$ be the pullback through $\rho_{V}$ of the orientation sheaf on $\mathbb{P}(V)$. Then there is an isomorphism

$$
2 H_{*}\left(\bar{Y}_{\mathcal{G}} ; \omega\right) \cong \begin{cases}0, & \operatorname{dim}(V) \bmod 2=0, \\ H_{(1)}^{\operatorname{dim}(V)-*}\left(\Pi_{\mathcal{G}}^{(2)} \cup\left\{V^{*}\right\}\right) \otimes \operatorname{or}(V), & \operatorname{dim}(V) \bmod 2=1 .\end{cases}
$$

In particular, if $\tilde{Y}_{\mathcal{G}}$ is the double cover over $\bar{Y}_{\mathcal{G}}$ associated to $\omega$, then

$$
H_{*}\left(\tilde{Y}_{\mathcal{G}} ; \mathbb{Z}[1 / 2]\right) \cong H_{*}\left(\bar{Y}_{\mathcal{G}} ; \omega \otimes \mathbb{Z}[1 / 2]\right) \oplus H_{*}\left(\bar{Y}_{\mathcal{G}} ; \mathbb{Z}[1 / 2]\right) .
$$

Proof (Sketch). The proof of the first claim simply follows the proof of Theorem 3.7, twisting by $\omega$ where appropriate. For the second claim, we observe that the short exact sequence

$$
0 \longrightarrow H_{*}\left(\bar{Y}_{\mathcal{G}} ; \mathbb{Z}[1 / 2]\right) \longrightarrow H_{*}\left(\tilde{Y}_{\mathcal{G}} ; \mathbb{Z}[1 / 2]\right) \longrightarrow H_{*}\left(\bar{Y}_{\mathcal{G}} ; \omega \otimes \mathbb{Z}[1 / 2]\right) \longrightarrow 0
$$

respects the grading by $\mathcal{C}_{\mathcal{G}}$, and in each graded component, either $H_{*}\left(\bar{Y}_{\mathcal{G}} ; \mathbb{Z}[1 / 2]\right)[A]$ or $H_{*}\left(\bar{Y}_{\mathcal{G}} ; \omega \otimes \mathbb{Z}[1 / 2]\right)[A]$ or both must vanish.

Another direction of potential generalization follows from the observation that the homology (modulo 2-torsion) depends only on the combinatorial data, namely the poset $\Pi_{\mathcal{G}}^{(2)}$. This suggests that there should be a more combinatorial construction of $\bar{Y}_{\mathcal{G}}$ itself. More precisely, it should be possible to generalize the main theorem (or even the mixed generalization considered above) to the case of a building set (of flats) in an oriented matroid (a combinatorial generalization of a real hyperplane arrangement, see [3]). In particular, the cell structure considered above seems particularly amenable to a description in oriented matroid terms, which would ideally give a proof that it actually corresponds to a CW complex. (Note in particular the fact that the proof of the Topological Representation Theorem (see, for example, $[\mathbf{3}$, Theorem 5.2.1]) attaches to any oriented matroid a natural regular cell decomposition of the sphere such that the antipode map is cellular. It should thus be straightforward to use this to construct analogs of De Concini-Procesi models in such a way as to agree with the geometric construction when the oriented matroid comes from an actual hyperplane arrangement.)

Finally, from a more algebraic perspective, it is worth noting that the condition that $\mathcal{G}$ be real is certainly not necessary for $\bar{Y}_{\mathcal{G}}$ to be defined over $\mathbb{R}$ and thus give rise to a smooth $\mathbb{R}$-manifold. Indeed, all that is truly necessary is that $\mathcal{G}$ be closed under complex conjugation. This has the effect of replacing certain pairs of real blow-ups by complex blow-ups, but the corresponding long exact sequences are still quite reasonable. The main difficulty is, once again, that the cell structure does not carry over, making it difficult to define the appropriate homology classes via a chain map. In addition, the action of the operad maps on homology is much more subtle, and in particular, need not respect the grading (even in the special case of $\bar{Y}_{\mathcal{G}}(\mathbb{C})$ viewed as a real manifold). 
We make the following conjecture in this case. Given an $\mathbb{R}$-rational building set $\mathcal{G}$, we say that a real subspace $C \in \mathcal{C}_{\mathcal{G}}$ is purely complex if its decomposition is of the form

$$
C=\bigoplus_{i} G_{i} \oplus \overline{G_{i}}
$$

Similarly, we denote by $\mathbb{R}(\mathcal{G})$ the subset of $\mathcal{G}$ consisting of real subspaces, and note that this is again a building set. Finally, for real $A \in \mathcal{C}_{\mathcal{G}}$, let $\mathbb{C}(A)$ denote the sum of the complex components of $A$.

Conjecture 1 . Let $\mathcal{G}$ be an $\mathbb{R}$-rational building set. Then $H_{*}\left(\bar{Y}_{\mathcal{G}}(\mathbb{R}), \mathbb{Z}\right)$ is naturally graded by $\mathbb{R}\left(\mathcal{C}_{\mathcal{G}}\right)$, and there is an isomorphism

$$
2 H_{*}\left(\bar{Y}_{\mathcal{G}}(\mathbb{R}), \mathbb{Z}\right)[A] \cong \bigoplus_{\substack{\mathbb{C}(A) \subset C \subset A \\ C \text { purely complex }}} H_{*}\left(\bar{Y}_{\left.\mathcal{G}\right|_{C}}(\mathbb{R}), \mathbb{Z}\right)[C] \otimes 2 H_{*}\left(\bar{Y}_{\mathbb{R}(\mathcal{G} / C)}(\mathbb{R}), \mathbb{Z}\right)[A / C] .
$$

REMARK. Note that $\bar{Y}_{\left.\mathcal{G}\right|_{C}}$ is (as a real algebraic variety) the restriction of scalars of a complex De Concini-Procesi model, so topologically is homeomorphic to that complex variety. In particular, the homology of its real locus is free; see [18] or the remark following Proposition 6.2 above for an explicit description of a basis.

Acknowledgements. The author would like to thank his coauthors P. Etingof, A. Henriques, and J. Kamnitzer on [7] for introducing him to these questions, and especially Henriques for discussions relating to blow-ups in $\mathbb{Z}[1 / 2]$-cohomology. In addition, the author would like to thank S. Devadoss and especially S. Yuzvinsky for motivating discussions, as well as several referees for useful comments.

\section{References}

1. S. M. Armstrong, M. Carr, S. L. Devadoss, E. Engler, A. Leininger and M. Manapat, 'Particle configurations and Coxeter operads', J. Homotopy Relat. Struct. 4(1) (2009) 83-109.

2. S. Axelrod and I. M. Singer, 'Chern-Simons perturbation theory. II', J. Differential Geom. 39 (1994) $173-213$.

3. A. Buörner, M. Las Vergnas, B. Sturmfels, N. White and G. M. Ziegler, Oriented matroids, 2nd edn, Encyclopedia of Mathematics and its Applications 46 (Cambridge University Press, Cambridge, 1999).

4. C. De Concini and C. Procesi, 'Wonderful models of subspace arrangements', Selecta Math. (N.S.) 1 (1995) 459-494.

5. P. Deligne, M. Goresky and R. MacPherson, 'L'algèbre de cohomologie du complément, dans un espace affine, d'une famille finie de sous-espaces affines', Michigan Math. J. 48 (2000) 121-136.

6. E. Delucchi, 'Subdivision of complexes of $k$-trees', Preprint, 2005, arXiv:math.CO/0509378.

7. P. Etingof, A. Henriques, J. Kamnitzer and E. M. Rains, 'The cohomology ring of the real locus of the moduli space of stable curves of genus 0 with marked points', Ann. of Math. (2) 171 (2010) 731-777.

8. E. M. Feichtner and I. Müller, 'On the topology of nested set complexes', Proc. Amer. Math. Soc. 133 (2005) 999-1006 (electronic).

9. G. GAiffi, 'Real structures of models of arrangements', Int. Math. Res. Not. 2004 (2004) 3439-3467.

10. M. Goresky and R. MacPherson, Stratified Morse theory, Ergebnisse der Mathematik und ihrer Grenzgebiete (3) 46 (Springer, Berlin, 1988).

11. A. Henderson and E. Rains, 'The cohomology of real De Concini-Procesi models of Coxeter type', Int. Math. Res. Not. 2008 (2008) article ID rnn001.

12. M. M. Kapranov, 'The permutoassociahedron, Mac Lane's coherence theorem and asymptotic zones for the KZ equation', J. Pure Appl. Algebra 85 (1993) 119-142.

13. S. KeEL, 'Intersection theory of moduli space of stable $n$-pointed curves of genus zero', Trans. Amer. Math. Soc. 330 (1992) 545-574.

14. V. A. Krasnov, 'Real algebraically maximal varieties', Math. Notes 73 (2003) 806-812.

15. G. I. Lehrer, 'On the Poincaré series associated with Coxeter group actions on complements of hyperplanes', J. London Math. Soc. (2) 36 (1987) 275-294.

16. M. DE Longueville and C. A. Schultz, 'The cohomology rings of complements of subspace arrangements', Math. Ann. 319 (2001) 625-646. 
17. A. Robinson, 'Partition complexes, duality and integral tree representations', Algebr. Geom. Topol. 4 (2004) 943-960 (electronic).

18. S. Yuzvinsky, 'Cohomology bases for the De Concini-Procesi models of hyperplane arrangements and sums over trees', Invent. Math. 127 (1997) 319-335.

19. S. Yuzvinsky, 'Small rational model of subspace complement', Trans. Amer. Math. Soc. 354 (2002) 1921-1945 (electronic).

Eric M. Rains

Department of Mathematics

University of California

Davis, $C A$

USA

Current address:

Department of Mathematics

California Institute of Technology

Pasadena, $C A$

USA

rains@caltech.edu 